\title{
Tight Regret Bounds for Noisy Optimization of a Brownian Motion
}

\author{
Zexin Wang, Vincent Y. F. Tan, Senior Member, IEEE, and Jonathan Scarlett, Member, IEEE
}

\begin{abstract}
We consider the problem of Bayesian optimization of a one-dimensional Brownian motion in which the $T$ adaptively chosen observations are corrupted by Gaussian noise. We show that as the smallest possible expected cumulative regret and the smallest possible expected simple regret scale as $\Omega(\sigma \sqrt{T / \log (T)}) \cap \mathcal{O}(\sigma \sqrt{T} \cdot \log T)$ and $\Omega(\sigma / \sqrt{T \log (T)}) \cap$ $\mathcal{O}(\sigma \log T / \sqrt{T})$ respectively, where $\sigma^{2}$ is the noise variance. Thus, our upper and lower bounds are tight up to a factor of $\mathcal{O}\left((\log T)^{1.5}\right)$. The upper bound uses an algorithm based on confidence bounds and the Markov property of Brownian motion (among other useful properties), and the lower bound is based on a reduction to binary hypothesis testing.
\end{abstract}

Index Terms-Bayesian optimization, Brownian motion, nonsmooth optimization, continuum-armed bandits, regret bounds, information-theoretic limits.

\section{INTRODUCTION}

Brownian motion (BM) is a continuous-time stochastic process widely studied in diverse fields such as physics [1], biology [2] and finance [3]. Specifically, BM is used to model random behavior of the movements of random particles in physical and biological systems, as well as the movements of financial asset prices. As a Gauss-Markov process, it inherits properties of a Gaussian process (GP), and also the Markov property. There have been several studies on methods and algorithms for optimizing a BM [4]-[7]. However, the fundamental limits (i.e., upper and lower bounds on the regret) for doing so have remained elusive for the most part; this is the main purpose of the present study. In the broader context of GPs, Bayesian optimization (BO) [8] is a sequential design strategy for global optimization of black-box functions. Here, a Gaussian process prior is assumed on an unknown function $f$. This prior is then updated to a posterior upon the observation of noisy samples, and further samples are selected based on the updated posterior. The high-level goal is to maximize $f$ in as few function evaluations as possible. More precisely, following existing works in the literature [9], [10], we consider the following performance metrics, which are respectively termed the simple regret and cumulative regret:

$$
r_{T}=\max _{x} f(x)-f\left(x^{(T)}\right)
$$

This work was supported in part by the Singapore National Research Foundation (NRF) under grant numbers R-252-000-A74-281 and R-263-000D02-281.

Z. Wang is with the Department of Mathematics, Imperial College London, London, SW7 2AZ, UK (email: zexin.wang19@imperial.ac.uk).

V. Y. F. Tan is with the Department of Electrical and Computer Engineering, and with the Department of Mathematics, National University of Singapore, Singapore (email: vtan@nus.edu.sg).

J. Scarlett is with the Department of Computer Science and with the Department of Mathematics, National University of Singapore, Singapore (email: scarlett@comp.nus.edu.sg).

$$
R_{T}=\sum_{t=1}^{T}\left(\max _{x} f(x)-f\left(x_{t}\right)\right) .
$$

Here, $x_{t}$ is the point chosen at time $t$, and $x^{(T)}$ is an additional point returned after the $T$-th time instant. It is important to note that $r_{T}$ and $R_{T}$ are random variables; they depend on the random function $f$, the noise introduced to the samples, and any source of algorithmic randomness in the selection of the points $\left\{x_{t}\right\}$.

The existing literature on BO focuses mainly on functions $f$ that are smooth (i.e., differentiable). However, in recent applications, non-smooth functions have become increasingly important [11]. Furthermore, gradient (or subgradient) information is often expensive or unavailable, and hence, zerothorder methods, motivating the study of $\mathrm{BO}$, are particularly attractive. We use BM as an archetypal example of a nonsmooth random function, and study the fundamental performance limits of BO on a standard BM. To do so, we consider both achievability results (existence results upper bounding the regret) and impossibility results (algorithm-independent lower bounds on the regret). For the former, we propose an asymptotically near-optimal algorithm based on upper and lower confidence bounds. For the latter, and for the latter we reduce the $\mathrm{BO}$ problem to a binary hypothesis test (see Sections $\mathrm{I}-\mathrm{A}$ and $\mathrm{I}-\mathrm{C}$ for related works).

Some potential applications of noisy BM optimization are as follows:

1) Bayesian optimization has been applied to environmental monitoring (e.g., see Marchant and Ramos [12]), which is inherently noisy due to imperfect sensors. In this context, erratic signals may be better modeled by BM compared to using smooth kernels.

2) Similarly, in hyperparameter tuning problems, erratic behavior may be modeled by a BM. For instance, in Swersky et al. [13], the closely-related non-smooth OrnsteinUhlenback process was adopted as part of the model.

Beyond any specific applications, we believe that this problem is important in the broad context of Bayesian optimization and continuous-armed or continuum-armed bandits. In particular, Scarlett [10] advanced the theory of smooth Bayesian optimization, but left a significant gap concerning non-smooth functions that our work partially closes.

\section{A. Related Work}

There have been several optimization algorithms proposed for BM in the literature; the most relevant one is by Grill et al. [4]. The main contribution therein is the proposal of an algorithm - termed Optimistic Optimization of a Brownian 
(OOB) - for the maximization of a BM in the noiseless setting. The sample complexity was shown to be $\mathcal{O}\left(\log ^{2}(1 / \varepsilon)\right)$. That is, the minimum number of samples to guarantee that one of the selected samples is $\varepsilon$-close to the global maximum of the BM with probability at least $1-\varepsilon$ is $\mathcal{O}\left(\log ^{2}(1 / \varepsilon)\right)$. This significantly improves over the earlier results of Al-Mharmah and Calvin [5] and Calvin et al. [7], in which the upper bound on the sample complexity was a polynomial in $1 / \varepsilon$.

There is also a vast literature on BO for smooth functions. In particular, for a GP with the squared-exponential (SE) kernel or the Matérn kernel with parameter $\nu>2$, Scarlett [10] gave cumulative regret bounds that are tight up to a $\sqrt{\log T}$ factor. Earlier bounds were also given for the GP-UCB algorithm in the prominent work of Srinivas et al. [9], attaining a near-tight upper bound for the SE kernel but not the Matérn kernel. In the noiseless case, Grünewälder et al. [14] gave both upper and lower cumulative regret bounds assuming the mean and kernel satisfy an $\alpha$-Hölder continuity condition, and Kawaguchi et al. [15] proved exponential convergence of the simple regret under certain smoothness assumptions on the kernel.

While our focus is in Bayesian optimization with a stochastic process model, there also exist several related works on the black-box optimization of deterministic functions with Höldertype continuity assumptions. For instance, see Munos [16] for the noiseless setting and Shang et al. [17] for the noisy setting, as well as the references therein. Connections between $\mathrm{BM}$ optimization and these works is further discussed in Section I-C below.

\section{B. Contributions}

Our main results state that the smallest possible expected cumulative regret for optimization of a BM with a fixed noise variance $\sigma^{2}>0$ behaves as $\Omega(\sigma \sqrt{T / \log (T)}) \cap$ $\mathcal{O}(\sigma \sqrt{T} \cdot \log T)$, and the smallest possible expected simple regret behaves as $\Omega(\sigma / \sqrt{T \log (T)}) \cap \mathcal{O}(\sigma \log T / \sqrt{T})$. In both cases, the gap between the upper and lower bound is only $\mathcal{O}\left((\log T)^{1.5}\right)$. In more detail, our technical contributions and observations consist of the following:

1) We develop an upper and lower confidence bound based algorithm that is amenable to the setting of noisy observations; this is in contrast to the OOB algorithm of Grill et al. [4], which is tailored to the noiseless setting. We characterize the performance in terms of both the simple regret and cumulative regret.

2) Our lower bound is based on a novel adaptation of the approach of [10], requiring several additional technical challenges to move from smooth functions to BM.

3) Our lower bound on the simple regret implies that the optimal sample complexity for the noisy setting is at least polynomial in $1 / \varepsilon$ for a fixed precision $\varepsilon$. This is much larger than the logarithmic upper bound for the noiseless counterpart proposed in [4].

4) The similarities between the cumulative 1 regret bounds and the corresponding proof techniques of $\mathrm{BO}$ for smooth

\footnotetext{
${ }^{1}$ In [10], simple regret was not considered, and in fact, attaining matching upper and lower simple regret bounds in the smooth setting appears to be more challenging due to subtleties regarding $O\left(\frac{1}{\sqrt{T}}\right)$ vs. $O\left(\frac{1}{T}\right)$ dependence $[18$.
}

functions [10] and BM indicate that the level of smoothness is not necessarily the key feature that dictates the difficulty of optimization, and that Hölder-type continuity may be similarly beneficial.

\section{Discussion of Existing Approaches}

We briefly pause to discuss certain approaches that one might consider adopting based on existing works, and comment on why they appear to be insufficient for the purposes of establishing our main results.

As mentioned above, Srinivas et al. [9] analyze the GPUCB algorithm, and provide an upper bound on the regret for general kernels in terms of a mutual information quantity called the information gain. While it is tempting to try to apply this result to Brownian motion, there are two major difficulties in doing so:

- In [9, Thm. 2], it is assumed that the derivatives of the function are bounded with high probability, rendering the result inapplicable for nowhere-differentiable processes such as BM consider in this paper. In fact, the authors go on to conjecture that their result does not hold for such processes; see the end of [9, Section V.A] therein.

- Even if an analogous result were to hold for BM, attaining a $\sqrt{T}$ poly $(\log T)$ regret bound would require showing that the information gain behaves as poly $(\log T)$, which appears to be unlikely given that even the smoother Matérn kernel only has an $\widetilde{\mathcal{O}}\left(T^{c}\right)$ bound (with $c \in(0,1)$ depending on the smoothness parameter $\nu$ ) on its information gain.

The above-mentioned work by Shang et al. [17] provides general bounds for the black-box optimization of deterministic functions with Hölder-type continuity assumptions. This continuity is captured by variants of the near-optimality dimension [16], which roughly quantifies the volume of $\epsilon$-optimal points that exist in the limit as $\epsilon \rightarrow 0$ (cf. Definition 2). It was demonstrated in Grill et al. [4] that the BM exhibits a certain modified definition of the near-optimality dimension that considers the average number of $\epsilon$-optimal points (since the $\mathrm{BM}$ is stochastic). When the near-optimality dimension according to this modified definition is substituted into the bounds for the noiseless deterministic setting [16] (despite the mismatch in the underlying definitions), the resulting regret bounds for the noiseless setting [4], [16] indeed match up to constant factors. An analogous observation turns out to hold in the noisy setting, with our upper bound coinciding with [17] in the same way that [4] coincides with [16].

However, while the bounds may end up matching in this sense (upon replacing the near-optimality dimension with its analog concerning the average number of $\epsilon$-optimal points), to the best of our knowledge, existing results for the deterministic setting do not formally transfer to the stochastic setting. The algorithm that we use for our upper bound resembles those of [4], [16], [17], but requires careful modifications and an analysis that specifically exploits several properties of the BM, such as its Markovity and normal increments; see Section IV-B and Appendix A for other properties of the BM that we exploit.

Another approach that one might consider is to simply take the noiseless BM optimization algorithm of Munos [16] 
and repeatedly sample every point sufficiently many times so that we essentially have close-to-noiseless observations. However, this turns out to yield a suboptimal result. Roughly speaking, resampling enough times to bring the uncertainty down to $\eta$ requires at least $\Omega\left(\frac{1}{\eta^{2}}\right)$ repetitions, and the resulting cumulative regret then scales as $\Omega\left(\frac{1}{\eta^{2}}+\eta T\right)$. This expression is minimized by $\eta=\Theta\left(T^{-\frac{1}{3}}\right)$, thus yielding suboptimal $\mathcal{O}\left(T^{\frac{2}{3}}\right)$ behavior (for the cumulative regret). In contrast, we attain a near-optimal bound by carefully considering the tradeoff between the number of resampled points and how many iterations have passed. In particular, our algorithm uses fewer resampled points in the earlier iterations and more resampled points in the later iterations.

Our lower bound follows similar high-level steps to [10], which in turn builds on earlier works such as [19]. However, the details are very different due to the fact that [10] crucially exploits the twice-differentiability of the function, whereas the $\mathrm{BM}$ is almost surely non-differentiable everywhere.

\section{Paper Organization}

The rest of the paper is structured as follows. In Section III we formally describe the problem setup and state our objectives. In Section III, we describe the algorithm used, state the achievable regret bounds, and provide the core steps of the proof. In Section IV, we state the impossibility results and provide their core proof steps. In Section $\mathrm{V}$, we conduct numerical experiments to demonstrate that the expected cumulative regret indeed scales as roughly $\sqrt{T}$. In Section VI we conclude our discussion. In Section VII, we prove several technical results used in the earlier sections, with some details further relegated to the appendices (supplementary material).

\section{Problem SETUP}

Over a fixed time horizon $T$, we seek to sequentially optimize a realization of a standard BM $W=\left(W_{x}\right)_{x \in D}$ over the one-dimensional domain $D=[0,1]$; any finite interval can be transformed to this choice via shifting and re-scaling. Note that we envision $W$ as being generated by nature "in advance", i.e., prior to any samples being taken.

At time 1 , we select a single point $x_{1} \in D$ and observe a noisy sample $y_{1}=W_{x_{1}}+z_{1}$ where $z_{1} \sim \mathcal{N}\left(0, \sigma^{2}\right)$ for some noise variance $\sigma^{2}>0$. At time $t \in\{2, \ldots, T\}$, given the previously sampled points and their noisy function evaluations $\left\{\left(x_{\tau}, y_{\tau}\right)\right\}_{\tau=1}^{t-1}$, we query an additional point $x_{t} \in D$ and observe a noisy sample $y_{t}=W_{x_{t}}+z_{t}$, where $\left(z_{t}\right)_{1 \leq t \leq T} \stackrel{\text { i.i.d }}{\sim}$ $\mathcal{N}\left(0, \sigma^{2}\right)$. We measure the optimization performance using the expected simple regret $\mathbb{E}\left[r_{T}\right]$ and expected cumulative regrets $\mathbb{E}\left[R_{T}\right]$, according to the definitions in (1) and (2) with $f(x)=W_{x}$.

When viewed as a GP, $W$ has zero mean and a nonstationary kernel $\mathbb{E}\left[W_{x_{1}} W_{x_{2}}\right]=\min \left(x_{1}, x_{2}\right)$. The $\mathrm{BM}$ has many useful properties [20], notably including the Markov property and normal increments: For all $0 \leq x_{1} \leq x_{2},\left(W_{x_{2}}-\right.$ $\left.W_{x_{1}}\right) \Perp W_{x_{1}}$ and $\left(W_{x_{2}}-W_{x_{1}}\right) \sim \mathcal{N}\left(0, x_{2}-x_{1}\right)$. Some further useful properties are stated in Appendix A

In the following analyses, we use $c_{1}, c_{2}$, etc. to denote generic universal constants that may differ from line to line.

\section{UPPER BOUNDS}

In this section, we introduce a confidence-bound based algorithm (see Algorithm 11) and derive an upper bound on its regret. The idea is to sequentially discretize the search space, and rule out suboptimal points using confidence bounds, as is commonly done in bandit algorithms (e.g., see [21 Ch. 22]). The algorithm works in epochs, with each epoch containing fewer remaining points and discretizing at a finer scale; the subsequent analysis seeks to bound the number of points sampled per epoch, and thereby obtain the overall regret bounds. These bounds are formally stated as follows.

Theorem 1. For the problem of BM optimization with a noise variance $\sigma^{2}>0$ satisfying $\sigma^{2} \geq \frac{\tilde{c}}{T^{1-\zeta}}$ for some positive constants $(\tilde{c}, \zeta),{ }^{2}$ there exists an algorithm (Algorithm 1 ) achieving the following:

$$
\mathbb{E}\left[R_{T}\right]=\mathcal{O}\left(\sigma T^{\frac{1}{2}} \log T\right), \text { and } \mathbb{E}\left[r_{T}\right]=\mathcal{O}\left(\sigma T^{-\frac{1}{2}} \log T\right) .
$$

In the rest of the section, we describe the algorithm and present the proof (with many details deferred to Section VII and the supplementary materials).

\section{A. Description of our algorithm}

Algorithm 1] works in epochs, with each epoch sampling points restricted to a finer grid than the previous epoch. This bears some resemblance to OOB by Grill et al. [4], and similar ideas have also been used for the optimization of smooth functions, e.g., by de Freitas et al. [22], with the main difference being the rule for discarding suboptimal points.

Let $T_{h}$ be the number of samples taken up to and including the $h$-th epoch, and $t_{h}$ be the number of samples taken during the $h$-th epoch. Hence, $T_{h}=\sum_{h^{\prime}=1}^{h} t_{h^{\prime}}$. We respectively define the upper and lower confidence bound 3 with noisy observations from the first $h$ epochs as

$$
\begin{aligned}
& \operatorname{UCB}_{h}([a, b]) \triangleq \max \left(\bar{y}_{a}^{(h)}, \bar{y}_{b}^{(h)}\right)+\beta_{\delta}(b-a), \\
& \operatorname{LCB}_{h}([a, b]) \triangleq \min \left(\bar{y}_{a}^{(h)}, \bar{y}_{b}^{(h)}\right)-\beta_{\delta}(b-a),
\end{aligned}
$$

where $\bar{y}_{x}^{(h)}=\operatorname{Average}\left(\left\{y_{s} \mid 1 \leq s \leq T_{h}, x_{s}=x\right\}\right)$ is the average of all observations at point $x \in D$ until the $h$-th epoch,

$$
\eta_{\delta}(x) \triangleq \sqrt{\frac{5 x}{2} \ln \left(\frac{2}{x \delta}\right)}, \text { and } \alpha_{\delta}(x) \triangleq \sqrt{6 x \ln \left(\frac{1}{x \delta}\right)},
$$

and $\beta_{\delta}(x) \triangleq \eta_{\delta}(x)+\alpha_{\delta}(x)$. Here, $\eta_{\delta}(x)$ is a term that was used in Grill et al. [4], and represents inherent uncertainty that would exist even in the case of noiseless observations. The term $\alpha_{\delta}(x)$ is additionally introduced due to the fact that we require wider confidence bounds in the presence of noise. With these notations, our algorithm is shown in Algorithm 1

At the $h$-th epoch, all sampled points (either at the midpoint or at the ends of the candidate intervals) are sampled at

\footnotetext{
${ }^{2}$ This is a very mild assumption, since we are primarily interested in the case that $\sigma$ is constant with respect to $T$.

${ }^{3}$ We preset $\delta$ as the probability of failure for the confidence bounds as a function of $T$, assuming that $T$ is fixed and known. The case of an unknown time horizon $T$ can be handled using a standard doubling trick, e.g, see [10 Appendix A]
} 
$\overline{\text { Algorithm } 1 \text { Elimination-based algorithm for Brownian mo- }}$ tion optimization

Require: Domain $D$, time horizon $T$

1: Initial set of intervals: $\mathcal{I}_{0}=\{[0,1]\}$

2: Initialize time index $t=1$ and epoch number $h=0$

3: Sample at $x=1$ for $\left\lceil\sigma^{2}\right\rceil$ times

4: Preset $\delta=T^{-1}$ as the error probability

5: while $t \leq T$ do

6: Find candidate intervals:

$$
\mathcal{L}_{h+1}=\left\{I \mid I \in \mathcal{I}_{h}, \operatorname{UCB}_{h}(I) \geq \max _{I \in \mathcal{I}_{h}} \operatorname{LCB}_{h}(I)\right\}
$$

7: $\quad$ Slice up the intervals:

$$
\mathcal{I}_{h+1}=\left\{\left[a, \frac{a+b}{2}\right],\left[\frac{a+b}{2}, b\right] \mid[a, b] \in \mathcal{L}_{h}\right\}
$$

8: Find the corresponding set of points:

$$
\mathcal{J}_{h+1}=\bigcup\left\{\left\{a, \frac{a+b}{2}, b\right\} \mid[a, b] \in \mathcal{L}_{h}\right\}
$$

9: $\quad$ Increment $t$ by $n_{h}\left|\mathcal{J}_{h+1}\right|$.

10: $\quad$ If $t \leq T$, then sample each point in $\mathcal{J}_{h+1}$ for $n_{h}$ times, where

$$
n_{h}=\left\lceil\sigma^{2} 2^{h+1}\right\rceil .
$$

Otherwise, if $t>T$, then only sample until a total of $T$ samples have been taken.

11: $\quad$ Increment $h$ by 1

12: end while

13: For the simple regret criterion, return $x^{(T)}$ uniformly at random from the set of selected points $\left\{x_{1}, \ldots, x_{T}\right\}$.

least $n_{h}$ times, to ensure a certain confidence level on $W$ across different points in the same epoch ${ }^{4}$ The set $\mathcal{L}_{h}$ can be viewed as the collection of intervals that potentially contain the maximizer; the confidence bounds shrink as more points are observed, and accordingly the total area of $\mathcal{L}_{h}$ shrinks as $h$ increases.

\section{B. Auxiliary Lemmas}

We now define some high probability events. Firstly, we define the following event, which was introduced in [4, Definition 1]:

$$
\mathcal{C} \triangleq \bigcap_{h=0}^{\infty} \bigcap_{k=0}^{2^{h}-1}\left\{\sup _{x \in\left[\frac{k}{2^{h}}, \frac{k+1}{2^{h}}\right]} W_{x} \leq B_{\left[k / 2^{h},(k+1) / 2^{h}\right]}\right\},
$$

where $B_{[a, b]}=\max \left(W_{a}, W_{b}\right)+\eta_{\delta}(b-a)$, and $\eta_{\delta}$ is defined in 10 . This is the event that the BM $W$ does not exceed a prescribed amount beyond its end points of every dyadic interval of the form $\left\{0,1 / 2^{h}, 2 / 2^{h}, \ldots,\left(2^{h}-1\right) / 2^{h}, 1\right\}$. In addition, we find it convenient to further define several other events, stated as follows.

\footnotetext{
${ }^{4}$ To mitigate the effect of the noise, at each epoch, we resample the chosen point at least $n_{h}$ times. Re-sampling is convenient for the analyses, whereas in practice one could use a more conventional UCB-type algorithm that samples a different point at each time instant (e.g., Srinivas et al. [9]).
}

Definition 1. We define the event $\mathcal{M} \triangleq \mathcal{M}_{1} \cap \mathcal{M}_{2} \cap \mathcal{M}_{3} \cap \mathcal{M}_{4}$, where

$$
\begin{aligned}
& \mathcal{M}_{1} \triangleq \bigcap_{h=0}^{\infty} \bigcap_{k=0}^{2^{h}-1}\left\{\left|W_{\frac{k+1}{2^{h}}}-W_{\frac{k}{2^{h}}}\right| \leq \alpha_{\delta}\left(2^{-h}\right)\right\}, \\
& \mathcal{M}_{2} \triangleq \bigcap_{h=0}^{\infty} \bigcap_{k \in \mathcal{J}_{h}}\left\{\left|\bar{y}_{\frac{k}{2^{h}}}-W_{\frac{k}{2^{h}}}\right| \leq \alpha_{\delta}\left(2^{-h}\right)\right\}, \\
& \mathcal{M}_{3} \triangleq \bigcap_{h=0}^{\infty} \bigcap_{k \in \mathcal{J}_{h}}\left\{\max _{x \in I_{h, k}} W_{x} \leq \operatorname{UCB}_{h}\left(I_{h, k}\right)\right\}, \\
& \mathcal{M}_{4} \triangleq \bigcap_{h=0}^{\infty} \bigcap_{k \in \mathcal{J}_{h}}\left\{\min _{x \in I_{h, k}} W_{x} \geq \operatorname{LCB}_{h}\left(I_{h, k}\right)\right\} .
\end{aligned}
$$

The event $\mathcal{M}_{1}$ represents the fact that the BM evaluated at successive points in the set of dyadic rationals $\left\{0,1 / 2^{h}, 2 / 2^{h}, \ldots,\left(2^{h}-1\right) / 2^{h}, 1\right\}$ yields a difference of at most $\alpha_{\delta}\left(2^{-h}\right) ; \mathcal{M}_{2}$ represents the effect of averaging out the noise in the observations; and $\mathcal{M}_{3}$ and $\mathcal{M}_{4}$ are analogous to event $\mathcal{C}$, and characterize a form of Hölder continuity restricted to the dyadic partition. Following [4], we henceforth refer to these as proxy-Hölder conditions on $W$. As stated in Algorithm 1, $\mathcal{J}_{h}$ is the set of ends of intervals in the set $\mathcal{I}$ at the $h$-th epoch. The events depend on $\delta$ through the evaluations of $\alpha_{\delta}$ and $\eta_{\delta}$.

In the following, we present some preliminary results to upper bound the regret. The first lemma states a standard high probability upper bound for a normal distribution.

Lemma 1. For any $\delta>0$, a standard Gaussian random variable is upper bounded by $\sqrt{2 \ln (1 / \delta)}$ with probability at least $1-\delta$.

Proof. This high-probability upper bound follows from the standard (Chernoff) bound $Q(\alpha) \leq e^{-\alpha^{2} / 2}$, where $Q(\alpha)$ is the complementary $\mathrm{CDF}$ of an $\mathcal{N}(0,1)$ random variable.

Next, we provide a formal statement that $\mathcal{M}$ holds with high probability. The proof is given in Section VII-A, with the main step being to generalize the analysis of the proxy-Hölder event $\mathcal{C}$ defined in 11 to its noisy variant, $\mathcal{M}_{3} \cap \mathcal{M}_{4}$.

Lemma 2. For any $\delta \in\left(0, \frac{1}{3}\right)$, we have $\mathbb{P}[\mathcal{M}] \geq 1-\delta^{2}$, where $\mathcal{M}$ implicitly depends on $\delta$.

We now seek to demonstrate the exponential shrinkage of the upper bounds for regret as the epoch number increases. Lemma 3 is a standard result used in the study of algorithms that eliminate sub-optimal points based on confidence bounds, though our confidence bounds are defined on intervals instead of specific points in $D$.

Lemma 3. Fix $\kappa>0$, and assume that at time $t$, for all intervals $[a, b]$ within some family of intervals $\mathcal{L}$, it holds for all $x \in[a, b]$ that $\mathrm{LB}_{h}([a, b]) \leq W_{x} \leq \mathrm{UB}_{h}([a, b])$ for some bounds $\mathrm{UB}_{h}$ and $\mathrm{LB}_{h}$ satisfying $\left.\right|^{5} \max _{[a, b] \in \mathcal{L}} \mid \mathrm{UB}_{h}([a, b])-$ $\operatorname{LB}_{h}([a, b]) \mid \leq 2 \kappa$. Then all $[a, b] \in \mathcal{L}$ containing $a 4 \kappa$ -

\footnotetext{
${ }^{5} \mathrm{We}$ will only apply this result in the case that the maximum exists, but more generally a supremum could be used.
} 
suboptimal point $x$ (i.e., $\left.W_{x}<\max _{I \in \mathcal{L}}\left(\max _{x^{\prime} \in I} W_{x^{\prime}}\right)-4 \kappa\right)$ must also satisfy the following:

$$
\mathrm{UB}_{h}([a, b])<\max _{I \in \mathcal{L}} \operatorname{LB}_{h}(I) .
$$

Proof. We have

$$
\begin{aligned}
\mathrm{UB}_{t}([a, b]) & \leq \operatorname{LB}_{t}([a, b])+2 \kappa \\
& \leq W_{x}+2 \kappa \\
& <\max _{I \in \mathcal{L}}\left(\max _{x^{\prime} \in I} W_{x^{\prime}}\right)-2 \kappa \\
& \leq \max _{I \in \mathcal{L}} \operatorname{UB}_{t}(I)-2 \kappa \\
& \leq \max _{I \in \mathcal{L}} \operatorname{LB}_{t}(I),
\end{aligned}
$$

where (17) and 21] use the assumption of $2 \kappa$-separation, (18) and 20 use the assumed validity of the confidence bounds, and (19) uses the assumption of $4 \kappa$-suboptimality.

The following lemma expresses the upper bound on the regret of a single point as an exponentially decreasing function of the epoch number $h$, and is proved via Lemma 3

Lemma 4. Conditioned on event $\mathcal{M}$, each point $x_{t}$ sampled in the $h$-th epoch satisfies $f\left(x^{*}\right)-f\left(x_{t}\right) \leq 4 \kappa_{h}$, where $\kappa_{h}=$ $\frac{5}{2} \alpha_{\delta}\left(2^{-h}\right)+\eta_{\delta}\left(2^{-h}\right)$.

Proof. For each $h \in \mathbb{N}$ and $k \in\left\{0,1, \ldots, 2^{h}-1\right\}$, we have

$$
\begin{aligned}
& \mathrm{UCB}_{h}\left(\left[\frac{k}{2^{h}}, \frac{k+1}{2^{h}}\right]\right)-\mathrm{LCB}_{h}\left(\left[\frac{k}{2^{h}}, \frac{k+1}{2^{h}}\right]\right) \\
& =\left|\bar{y}_{\frac{k}{2^{h}}}^{(t)}-\bar{y}_{\frac{k+1}{2^{h}}}^{(t)}\right|+2 \alpha_{\delta}\left(2^{-h}\right)+2 \eta_{\delta}\left(2^{-h}\right) \\
& \leq\left|\bar{y}_{\frac{k}{2^{h}}}^{(t)}-W_{\frac{k}{2^{h}}}\right|+\left|\bar{y}_{\frac{k+1}{2^{h}}}^{(t)}-W_{\frac{k+1}{2^{h}}}\right|+\left|W_{\frac{k}{2^{h}}}-W_{\frac{k+1}{2^{h}}}\right| \\
& \quad+2 \alpha_{\delta}\left(2^{-h}\right)+2 \eta_{\delta}\left(2^{-h}\right) \\
& \leq 2\left[\frac{5}{2} \alpha_{\delta}\left(2^{-h}\right)+\eta_{\delta}\left(2^{-h}\right)\right]=2 \kappa_{h}
\end{aligned}
$$

where (22) uses the definitions of $\mathrm{UCB}_{h}$ and $\mathrm{LCB}_{h}$ in (8) (9), 23) follows from the triangle inequality, and (24) follows from the definitions of $\mathcal{M}_{1}$ and $\mathcal{M}_{2}$ in the definition of $\mathcal{M}$.

Since $\mathcal{M}$ implies that $W_{x}$ is sandwiched between $\mathrm{UCB}_{h}$ and $\mathrm{LCB}_{h}$, Lemma 3 implies that the regret for each point $x_{t}$ sampled in the $h$-th epoch is at most $4 \kappa_{h}$.

We restate a lemma from [4] regarding the expected number of near-optimal points. An $\eta$-near-optimal point $x$ is one with value $W_{x}$ that is $\eta$-close to $M:=\max _{x \in[0,1]} W_{x}$.

Definition 2. Let $\mathcal{N}_{h}(\eta)$ denote the number of $\eta$-near-optimal points among $\left\{0,2^{-h}, \ldots, 1\right\}$ :

$$
\mathcal{N}_{h}(\eta) \triangleq\left|\left\{k \in\left\{0,1, \ldots, 2^{h}\right\} \quad: W_{k / 2^{h}} \geq M-\eta\right\}\right| .
$$

Lemma 5 ( [4, Lemma 3]). The expected number of $\eta$-nearoptimal points $\mathcal{N}_{h}(\eta)$ in a $2^{-h}$-spaced grid in $[0,1]$ is upper bounded as follows:

$$
\mathbb{E}\left[\mathcal{N}_{h}(\eta)\right] \leq 6 \eta^{2} 2^{h}
$$

\section{Completion of the Proof of Theorem 1}

Let $r_{(h)}$ be the maximum instantaneous regret incurred in the $h$-th epoch, and recall that $T_{h}$ is the number of queries during the $h$-th epoch. Both are random variables, and we first analyze their behavior under event $\mathcal{M}$. By Lemma 4 , event $\mathcal{M}$ implies that

$$
r_{(h)} \leq 4 \kappa_{h},
$$

and in addition, the definition of $\kappa_{h}$ therein yields

$$
\begin{aligned}
\kappa_{h} & =\frac{c_{1}^{\prime \prime}}{\sqrt{2^{h}}}\left[\sqrt{\ln \left(\frac{2^{6 h}}{2 \pi \delta^{6}}\right)}+\sqrt{\ln \left(\frac{2^{h}}{\delta}\right)}\right] \\
& \leq c_{1} 2^{-\frac{h}{2}}(2 \sqrt{\ln (1 / \delta)}+\sqrt{h}) \\
& =c_{1} 2^{-\frac{h}{2}}(\sqrt{\ln T}+\sqrt{h}),
\end{aligned}
$$

where (28) uses the definitions of $\alpha_{\delta}$ and $\eta_{\delta}$ in (10), and (30) follows from the choice of $\delta=T^{-1}$. By an analogous argument, we also have the lower bound

$$
\kappa_{h} \geq c_{1}^{\dagger} 2^{-\frac{h}{2}}(\sqrt{\ln T}+\sqrt{h})
$$

for some $c_{1}^{\dagger}>0$.

Next, we introduce a quantity $h^{\prime}$ that can be viewed as approximately characterizing the average total number of epochs. In accordance with 27), it is natural to consider the choice $\eta=4 \kappa_{h}$ in Lemma 5 , which leads to a right-hand side of $96 \kappa_{h}^{2} 2^{h}$ in (26). Since each point is sampled at least $\sigma^{2} 2^{h}$ times in Algorithm 1, and the total number of samples in a given epoch can never exceed $T$, we should expect that the epoch index never exceeds the following:

$$
h^{\prime}=\max \left\{h: 96 \sigma^{2} \kappa_{h}^{2} 2^{2 h}<T\right\} .
$$

We emphasize that we do not require a formal claim relating the number of epochs to $h^{\prime}$, but we still find the preceding intuition useful.

We claim that there exist positive constants $c_{2}^{\dagger}, c_{2}^{\dagger \dagger}$, and $c_{2}^{\natural}$ such that the following bounds hold:

$$
\begin{aligned}
c_{2}^{\dagger} \frac{T}{\sigma^{2} \ln T} & \leq 2^{h^{\prime}} \leq c_{2}^{\dagger \dagger} \frac{T}{\sigma^{2} \ln T}, \\
h^{\prime} & \leq c_{2}^{\natural} \ln T .
\end{aligned}
$$

To see this, we consider the choice of $h$ such that the lower bound in (33) holds with equality, and study the left-hand term in (32) as follows:

$$
\begin{aligned}
96 \sigma^{2} \kappa_{h}^{2} 2^{2 h} & \leq 96 \sigma^{2} c_{1}^{2} 2^{h}(\sqrt{\ln T}+\sqrt{h})^{2} \\
& =96 c_{1}^{2} \frac{c_{2}^{\dagger} T}{\sigma^{2} \ln T}(\sqrt{\ln T}+\sqrt{h})^{2},
\end{aligned}
$$

where (35) uses (30), and (36) substitutes the left-hand side of (33). To eliminate the remaining $\sqrt{h}$ term, we recall the assumption $\sigma^{2} \geq \frac{c_{\sigma}}{T^{1-\zeta}}$ in Theorem 1, and note that taking the logarithm (base 2) of $2^{h}=c_{2}^{\dagger} \frac{T}{\sigma^{2} \ln T}$ gives $h=O\left(\ln T+\ln c_{2}^{\dagger}\right)$. Hence, 36 gives for sufficiently small $c_{2}^{\dagger}$ that $96 \sigma^{2} \kappa_{h}^{2} 2^{2 h}<$ $T$, and hence, this choice of $h$ is indeed a lower bound on $h^{\prime}$, as desired. The upper bound on $h^{\prime}$ follows from a near-identical argument, and we notice that 34 was already established as 
an intermediate step.

Let $\mathbb{E}_{\mathcal{M}}[\cdot]=\mathbb{E}[\cdot \mid \mathcal{M}]$ be the conditional expectation given the event $\mathcal{M}$. We now provide an upper bound on the conditional expected cumulative regret as follows, starting with 27) (recall also $n_{h}$ defined in Algorithm 11:

$$
\begin{aligned}
& \mathbb{E}_{\mathcal{M}}\left[R_{T}\right] \\
& \leq \sum_{h=1}^{h_{\max }} 4 \kappa_{h} \mathbb{E}_{\mathcal{M}}\left[t_{h}\right] \\
& \leq \sum_{h=1}^{\infty} 4 \kappa_{h} \min \left(n_{h} \mathbb{E}_{\mathcal{M}}\left[\left|\mathcal{J}_{h}\right|\right], T\right) \\
& \leq \sum_{h=1}^{\infty} 4 \kappa_{h} \min \left(n_{h} \mathbb{E}_{\mathcal{M}}\left[\mathcal{N}_{h+1}\left(4 \kappa_{h}\right)\right], T\right) \\
& \leq \sum_{h=1}^{\infty} 4 \kappa_{h} \min \left(\frac{96}{1-\delta^{2}} \kappa_{h}^{2} 2^{h+1}\left(\sigma^{2} 2^{h+1}+1\right), T\right) \\
& \leq \sum_{h=1}^{h^{\prime}} \frac{384}{1-\delta^{2}} \kappa_{h}^{3} 2^{h+1}\left(\sigma^{2} 2^{h+1}+1\right)+\sum_{h=h^{\prime}+1}^{\infty} 4 \kappa_{h} T \\
& \leq c_{2}^{\prime \prime} \sum_{h=1}^{h^{\prime}}\left(\sigma^{2} 2^{\frac{h}{2}}+2^{-\frac{h}{2}}\right)(\sqrt{\ln T}+\sqrt{h})^{3} \\
& \quad+c_{1} \sum_{h=h^{\prime}+1}^{\infty} T 2^{-\frac{h}{2}}(\sqrt{\ln T}+\sqrt{h}) \\
& \leq c_{2} \sigma \sqrt{T} \ln T \\
& \leq c_{2}^{\prime}\left[\sigma^{2} \max \left(\ln T, h^{\prime}\right)^{\frac{3}{2}} 2^{\frac{h^{\prime}}{2}}+(\ln T)^{\frac{3}{2}}\right. \\
& \left.\quad+T 2^{-\frac{h^{\prime}}{2}} \sqrt{\max \left(\ln T, h^{\prime}\right)}\right] \\
& \leq
\end{aligned}
$$

where:

- 38 follows from $t_{h} \leq T$ and the fact that each point in $\mathcal{J}_{h}$ is sampled $n_{h}$ times;

- 39 follows from Lemma 4 .

- 40 follows from Lemma 5 and $n_{h}=\left\lceil\sigma^{2} 2^{h+1}\right\rceil$, as well as the fact that for any random variable $A, \mathbb{E}_{\mathcal{M}}[A]=$ $\frac{\mathbb{E}[A \mathbf{1}\{\mathcal{M}\}]}{\mathbb{P}[\mathcal{M}]} \leq \frac{\mathbb{E}[A]}{1-\delta^{2}}$ (recall from Lemma 2 that $\mathbb{P}[\mathcal{M}] \geq$ $\left.1-\delta^{2}\right)$

- 41 follows by upper bounding the minimum by either of its two arguments (choosing which one differently depending on the summation index);

- 42 follows from (30);

- 43 follows from the fact that an exponentially decreasing (resp., increasing) series is bounded above by a constant multiple of its first (resp., last) term - the first two terms come from splitting $\left(\sigma^{2} 2^{\frac{h}{2}}+2^{-\frac{h}{2}}\right)$ from 42. to form two sums, the first of which is exponentially increasing, and the second of which is exponentially decreasing;

- (44) follows by substituting the bounds on $h^{\prime}$ from (33) (34), and also noting that the $(\ln T)^{\frac{3}{2}}$ term is insignificant compared to $\sigma \sqrt{T} \ln T$ due to the assumption $\sigma^{2} \geq \frac{c_{\sigma}}{T^{1-\zeta}}$ in Theorem 1

We can now upper bound the unconditional expectation of
$R_{T}$ via the law of total expectation as follows:

$$
\begin{aligned}
\mathbb{E}\left[R_{T}\right] & =\mathbb{E}_{\mathcal{M}}\left[R_{T}\right] \mathbb{P}[\mathcal{M}]+\mathbb{E}_{\mathcal{M}^{c}}\left[R_{T}\right] \mathbb{P}\left[\mathcal{M}^{c}\right] \\
& \leq c_{2} \sigma \sqrt{T} \ln T+\mathcal{O}\left(T \sqrt{\log (1 / \delta)} \delta^{2}\right) \\
& =\mathcal{O}(\sigma \sqrt{T} \log T),
\end{aligned}
$$

where (46) is established by showing that $\mathbb{E}\left[R_{T} \mid \mathcal{A}\right]=$ $\mathcal{O}\left(T \sqrt{\log \frac{1}{\mathbb{P}[\mathcal{A}]}}\right)$ for any event $\mathcal{A}$ (see Section VII-B for details) and applying $\mathbb{P}\left[\mathcal{M}^{c}\right] \leq \delta^{2}$ (see Lemma 2, and 47) follows from the fact that $\delta=T^{-1}$ and the assumption $\sigma^{2} \geq \frac{c_{\sigma}}{T^{1-\zeta}}$. This yields the first part of Theorem 1

In addition, by the choice of $x^{(T)}$ in the last line in Algorithm 1, the upper bound on the expected simple regret trivially follows:

$$
\mathbb{E}\left[r_{T}\right] \leq \frac{\mathbb{E}\left[R_{T}\right]}{T}=\mathcal{O}\left(\sigma T^{-\frac{1}{2}} \log T\right),
$$

which yields the second part of Theorem 1

\section{LOWER BOUNDS}

In this section, we establish algorithm-independent lower bounds on the regret. The idea of the proof is to reduce the optimization problem into a binary hypothesis testing problem, while confining attention to "typical" realizations of a Brownian motion by conditioning on realizations satisfying suitable high-probability properties (e.g., a proxy-Hölder type condition). Once the reduction to hypothesis testing is done, a lower bound is deduced via Fano's inequality. As hinted earlier, this proof strategy builds on that of [10] (and in turn [19]), but with very different details due to the consideration of BM instead of smooth and stationary functions.

We begin by formally stating our lower bounds.

Theorem 2. For the problem of BM optimization with a noise variance $\sigma^{2}>0$ satisfying $\sigma^{2} \leq \tilde{c}^{\prime} T^{1-\zeta^{\prime}}$ for some positive constants $\left(\tilde{c}^{\prime}, \zeta^{\prime}\right),{ }^{6}$ any algorithm must have

$$
\begin{aligned}
\mathbb{E}\left[r_{T}\right] & =\Omega\left(\sigma(T \log T)^{-\frac{1}{2}}\right), \quad \text { and } \\
\mathbb{E}\left[R_{T}\right] & =\Omega\left(\sigma(T / \log T)^{\frac{1}{2}}\right) .
\end{aligned}
$$

The proof is given in the remainder of the section, with several details deferred to Section VII-F

\section{A. Reduction to Binary Hypothesis Testing}

We fix $\Delta>0$, and view the BM $W$ on $D=[0,1]$ as being generated by the following procedure:

1) Generate a BM $\tilde{W}$ on the larger domain $[-\Delta, 1+\Delta]$, with $\tilde{W}_{-\Delta}=0$ (i.e., $\tilde{W}$ is shifted left by $\Delta$ compared to a standard BM).

2) Randomly draw $V \in\{+,-\}$ with probability $\frac{1}{2}$ each, and perform one of the following steps to generate $\tilde{W}^{\prime}$ :

- If $V$ is ' + ', then shift $\tilde{W}$ left along the $x$-axis by $\Delta$, and add a constant term of $-\tilde{W}_{\Delta}$.

- If $V$ is '-', then shift $\tilde{W}$ right along the $x$-axis by $\Delta$.

${ }^{6}$ This is a very mild assumption, since we are primarily interested in the case that $\sigma$ is constant with respect to $T$. 


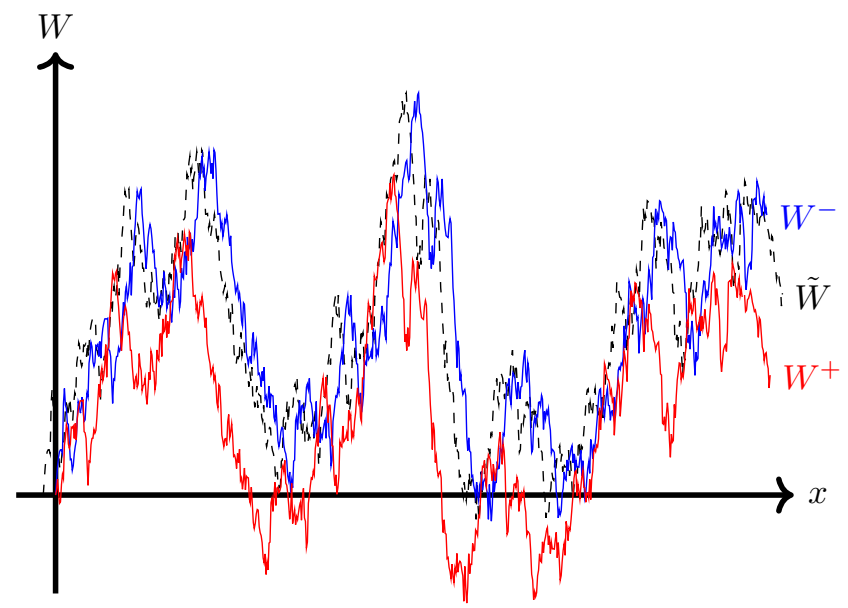

Fig. 1: Illustration of $\tilde{W}, W^{+}$and $W^{-}$

The two corresponding functions are written as

$$
\begin{aligned}
& W_{x}^{+}=\tilde{W}_{x+\Delta}-\tilde{W}_{\Delta}, \\
& W_{x}^{-}=\tilde{W}_{x-\Delta},
\end{aligned}
$$

and we observe that $W_{0}^{+}=W_{0}^{-}=0$, and by the Markov property, both $W_{0}^{+}$and $W_{0}^{-}$are standard BM processes when restricted to the domain $[0,1]$. We allow $\Delta$ to vary with $T$, and will in fact eventually set $\Delta=\mathcal{O}(1 / T)$.

3) Let $W_{x}=\tilde{W}_{x}^{\prime}$ for $x \in[0,1]$. Since $W_{x}^{+}$and $W_{x}^{-}$are both standard BM, we have that $W_{x}$ is a standard BM conditioned on either value of $V$, and thus, it is also a standard BM unconditionally, as desired.

We consider a "genie-aided" argument in which $\tilde{W}$ is revealed to the algorithm but the direction of the shift (i.e., the value of $V$ ) is unknown. Clearly this additional information provided by the genie can only help the algorithm, so any lower bound still remains valid for the original setting. Stated differently, the algorithm knows that $W$ is either $W^{+}$or $W^{-}$. This argument allows us to reduce the BO problem to a binary hypothesis test with adaptive sampling. The hypothesis, indexed by $v \in\{+,-\}$, is that the underlying function is $W^{v}$.

We define the maximizer of $\tilde{W}$ as

$$
x_{M}=\underset{x \in[-\Delta, 1+\Delta]}{\arg \max } \tilde{W}_{x}
$$

(which is almost surely unique), and let

$$
x_{M}^{+}=x_{M}-\Delta, \quad \text { and } \quad x_{M}^{-}=x_{M}+\Delta .
$$

Then, the maxima of the processes $W_{x}^{-}$and $W_{x}^{+}$are respectively defined as

$$
\begin{aligned}
& M^{-}:=W_{x_{M}^{-}}^{-}=\tilde{W}_{x_{M}}=M, \\
& M^{+}:=W_{x_{M}^{+}}^{+}=M-\tilde{W}_{\Delta},
\end{aligned}
$$

and we define the following functions that, up to a caveat discussed below, represent the (simple and cumulative) regret with respect to $W^{+}$and $W^{-}$:

$$
r^{+}(x)=M^{+}-W_{x}^{+}, \quad r^{-}(x)=M^{-}-W_{x}^{-},
$$

$$
R_{T}^{+}=\sum_{t=1}^{T} r^{+}\left(x_{t}\right), \quad R_{T}^{-}=\sum_{t=1}^{T} r^{-}\left(x_{t}\right) .
$$

It is important to note that $x_{M}^{+}$and $x_{M}^{-}$in 54 could, in principle, lie outside the domain $[0,1]$ (namely, when $x_{M}<\Delta$ or $x_{M}>1-\Delta$ ), in which case it may hold that the simple regret $r_{T}$ satisfies $r_{T}<r_{T}^{v}$ for some $v \in\{+,-\}$. However, in our analysis, we will condition on a high-probability event (see Definition 3 that ensures $x_{M}^{+}, x_{M}^{-} \in[0,1]$, and conditioned on this event we have $r_{T}=r_{T}^{V}$. Similar observations apply for the cumulative regret.

\section{B. Auxiliary Lemmas}

We first state some useful properties of a BM. The Brownian meander [23] plays an important role in our analysis, as it characterizes the distribution of function values to the left and right of the maximum of $W$ (see Lemma A.4 in Appendix A). Formally, given a standard BM $\left(W_{x}\right)_{x \in[0,1]}$, we define a Brownian meander as the process $\left(W_{x} \mid \min _{x^{\prime} \in[0,1]} W_{x^{\prime}} \geq 0\right)$ for $x \in[0,1]$. Note that although the event $\left\{\min _{x^{\prime} \in[0,1]} W_{x^{\prime}} \geq 0\right\}$ has probability zero, this conditional distribution is known to remain well-defined [23].

The following lemmas characterize the distribution of the running maximum or minimum of a Brownian meander; the proofs are given in Sections VII-C and VII-D

Lemma 6. For a standard $B M W$, for any $0<s<t$ and $0 \leq x<\frac{\sqrt{s}}{2}$, it holds that

$$
\begin{aligned}
& \mathbb{P}\left[\max _{0 \leq z \leq s} W_{z} \geq x \mid \min _{0<z \leq t} W_{z}>0, W_{0}=0\right] \\
& \quad \geq\left\{\begin{array}{cl}
1-\frac{1}{2}\left(\frac{x}{\sqrt{s}}\right)^{2} & \text { if } t>2 s \\
1-\frac{x \sqrt{2}}{\sqrt{s}} & \text { if } t \leq 2 s .
\end{array}\right.
\end{aligned}
$$

Lemma 7. For a $B M W$ with initial value $W_{0}=u$, we have for any $0<\varepsilon<u$ that

$$
\mathbb{P}\left[\min _{0 \leq z \leq t} W_{z}>\varepsilon \mid \min _{0 \leq z \leq t} W_{z}>0, W_{0}=u\right] \geq \frac{u-\varepsilon}{u} .
$$

We define a high-probability event to restrict the position of the maximum to the interval $(2 \Delta, 1-2 \Delta)$, and to restrict the two regret functions $r^{+}$and $r^{-}$to be simultaneously lower than a certain function of $\Delta$. Note that the constant $\delta>0$ in the following is not related to that appearing in Section III.

Definition 3. Fix $\delta>0$, and let $\mathcal{T} \triangleq \mathcal{T}_{1} \cap \mathcal{T}_{2} \cap \mathcal{T}_{3}$, where

$$
\begin{aligned}
& \mathcal{T}_{1} \triangleq\left\{2 \Delta<x_{M}<1-2 \Delta\right\}, \\
& \mathcal{T}_{2} \triangleq\left\{\forall x \in D, \max \left(r^{+}(x), r^{-}(x)\right) \geq c_{3} \delta^{2} \sqrt{\Delta}\right\}, \\
& \mathcal{T}_{3} \triangleq\left\{\forall x \in D,\left|r^{+}(x)-r^{-}(x)\right| \leq c_{4} \sqrt{\Delta \ln (1 / \Delta)}\right\} .
\end{aligned}
$$

As the following lemma is crucial, we outline the proof here, and provide the full details in Section VII-E

Lemma 8. For $0<\delta<1$, and any $0<\eta<\frac{1}{2}$ and sufficiently small $\Delta$, we have

$$
\mathbb{P}[\mathcal{T}] \geq 1-3 \Delta^{\eta}-\delta-\Delta
$$

where $\mathcal{T}$ implicitly depends on $\delta$. 


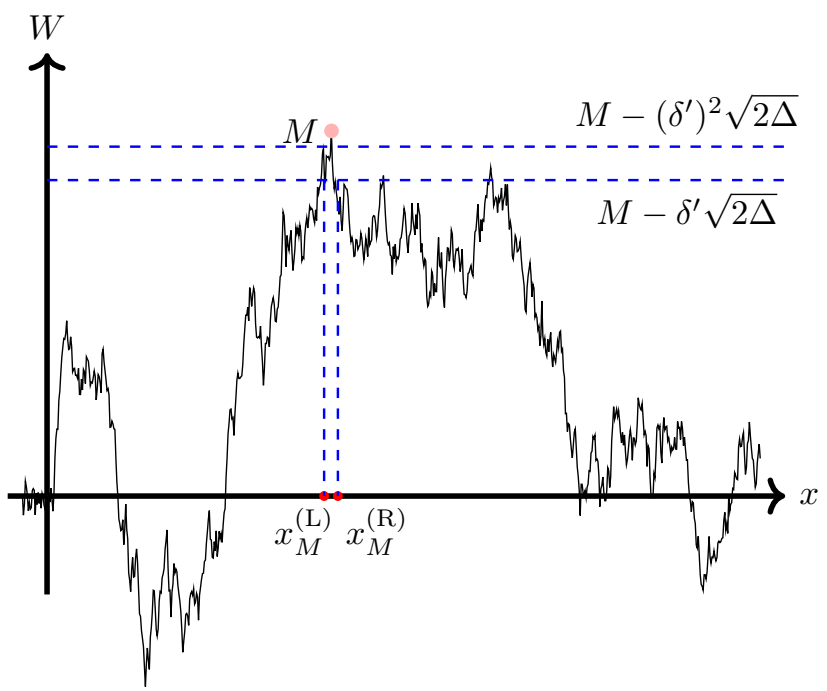

Fig. 2: Running maxima of the Brownian motion outside the neighborhood near its maximum

Proof Sketch of Lemma 8 The probability of $\mathcal{T}_{1}$ is characterized via a direct analysis of the probability of the maximum lying in the intervals $[0,2 \Delta]$ and $[1-2 \Delta, 1]$. In the limit of small $\Delta$, we show that the latter probability decays to zero as roughly $\tilde{O}(\Delta)$ (up to logarithmic factors), and hence, when $\Delta$ is sufficiently small, $\mathcal{T}_{1}$ holds with probability at least $1-\Delta^{\eta}$.

For events $\mathcal{T}_{2}$ and $\mathcal{T}_{3}$, we consider the right-most time $x_{M}^{(\mathrm{L})}$ left of the maximizer $x_{M}$ to hit the value $M-\delta^{\prime} \sqrt{2 \Delta}$, where $\delta^{\prime}$ is a constant multiple of $\delta$. With probability at least $1-\left(\delta^{\prime}\right)^{2}, x_{M}^{(\mathrm{L})}$ falls within the $\Delta$-neighborhood of $x_{M}$ by Lemma 6 , a result concerning the running maximum of a Brownian meander. In addition, we can lower bound the probability of the running maximum from 0 to $x_{M}^{(\mathrm{L})}$ exceeding $M-\left(\delta^{\prime}\right)^{2} \sqrt{2 \Delta}$ by $1-\delta^{\prime}$ by Lemma 7 , a result concerning the running minimum of a Brownian meander starting from a non-zero value to reach certain lower value.

As illustrated in Figure 2, we define two more "mirror events" of these two on the right of $x_{M}$. When $\mathcal{T}_{1}$ and these four events simultaneously hold, we have that all values larger than $M-\left(\delta^{\prime}\right)^{2} \sqrt{2 \Delta}$ must be in the $\Delta$-neighborhood of $x_{M}$ for either of $W^{+}$or $W^{-}$. Furthermore, with the horizontal shift of $2 \Delta$, the $\Delta$-neighborhoods near the maximum of the two functions do not overlap, and the shift would not result in a maximum outside $[0,1]$. By taking a union bound on all of these events, $\mathcal{T}_{1} \cap \mathcal{T}_{2}$ holds with probability at least $1-3 \Delta^{\eta}-\delta$ when we let $c_{3} \delta^{2} \sqrt{\Delta}=\left(\delta^{\prime}\right)^{2} \sqrt{2 \Delta}$, where $c_{3}=0.01 \sqrt{2}$.

The analysis of event $\mathcal{T}_{3}$ uses similar ideas to the analysis of event $\mathcal{M}$ in Lemma 2 Recall that $r^{+}$and $r^{-}$are shifted versions of each other, and the shift along the horizontal axis is $2 \Delta$. A standard proxy-Hölder continuity argument (e.g., see [4]) can be used to establish that all points separated by $2 \Delta$ have corresponding $\tilde{W}_{x}$ values differing by $\mathcal{O}(\sqrt{\Delta \ln (1 / \Delta)})$ (uniformly on the domain $D$ ) with high probability; a more quantitative version of this argument yields $\mathbb{P}\left[\mathcal{T}_{3}\right] \geq 1-\Delta$.

To establish lower bounds for the expected cumulative regret and simple regret, we make use of Fano's inequality [24], which naturally introduces the mutual information between the hypothesis $V$ and the selected points and observations $(\mathbf{x}, \mathbf{y})$, where $\mathbf{x}=\left(x_{1}, \ldots, x_{T}\right)$ and $\mathbf{y}=\left(y_{1}, \ldots, y_{T}\right)$ with $\mathbf{x} \in[0,1]^{T}$ and $\mathbf{y} \in \mathbb{R}^{T}$. In the following, we will also condition on $\{\tilde{W}=\tilde{w}\}$, where $\tilde{w}$ is a specific realization of $\tilde{W}$ satisfying the conditions in $\mathcal{T}$ (Definition 3). For brevity, this conditioning is indicated as a subscript $\tilde{w}$. For example, we write $I_{\tilde{w}}(V ; \mathbf{x}, \mathbf{y})$ as a shorthand for the conditional mutual information $I(V ; \mathbf{x}, \mathbf{y} \mid \tilde{W}=\tilde{w})$. Recall that $V$ is assumed to be equiprobable on $\{+,-\}$.

Lemma 9. Under the preceding setup with $\tilde{w}$ satisfying the conditions in $\mathcal{T}$, we have

$$
\mathbb{E}_{\tilde{w}}\left[r_{T}\right] \geq c_{3} \delta^{2} \sqrt{\Delta} H_{2}^{-1}\left(\ln 2-I_{\tilde{w}}(V ; \mathbf{x}, \mathbf{y})\right)
$$

where $H_{2}^{-1}:[0, \log 2] \rightarrow[0,1 / 2]$ is the functional inverse of the binary entropy function $H_{2}(\alpha)=\alpha \ln (1 / \alpha)+(1-$ $\alpha) \ln (1 /(1-\alpha))$ in nats.

Proof. The proof mostly follows Raginsky and Rakhlin [19] and Scarlett [10] (and related earlier works on statistical estimation), and can be found in Section VII-F.

\section{Completion of the Proof of Theorem 2}

We upper bound mutual information $I_{\tilde{w}}(V ; \mathbf{x}, \mathbf{y})$ conditioned on $\tilde{W}=\tilde{w}$ satisfying the conditions defining event $\mathcal{T}$ :

$$
\begin{aligned}
I_{\tilde{w}}(V ; \mathbf{x}, \mathbf{y}) & \leq \sum_{t=1}^{T} I_{\tilde{w}}\left(V ; y_{t} \mid x_{t}\right) \\
& \leq \sum_{t=1}^{T} \max _{x \in[0,1]} \frac{\left(r^{+}(x)-r^{-}(x)\right)^{2}}{2 \sigma^{2}} \\
& \leq \frac{c_{4}^{2}\left(\Delta \ln \frac{1}{\Delta}\right) T}{2 \sigma^{2}}
\end{aligned}
$$

where (66) follows from the tensorization property of mutual information [24, Lemma 3], 677) follows from a standard calculation of relative entropy between Gaussian random variables (and replacing the average over $x_{t}$ by a maximum over $x \in[0,1]$ ), and (68) follows from event $\mathcal{T}_{3}$ in Definition 3 .

To ensure that $H_{2}^{-1}\left(\ln 2-I_{\tilde{w}}(V ; \mathbf{x}, \mathbf{y})\right)$ is lower bounded by a positive constant, we choose $\Delta=c_{4}^{\prime} \frac{\sigma^{2}}{T \ln T}$ for some $c_{4}^{\prime}>0$. The assumption $\sigma^{2} \leq \tilde{c}^{\prime} T^{1-\zeta^{\prime}}$ in Theorem 2 ensures that $\ln \frac{1}{\Delta}=\Theta(\log T)$; hence, the $\ln \frac{1}{\Delta}$ and $\ln T$ terms cancel upon substitution into 68 , and we obtain $I_{\tilde{w}}(V ; \mathbf{x}, \mathbf{y}) \leq \frac{1}{2}$ (say) for sufficiently small $c_{4}^{\prime}$. The resultant inequality of 65. therefore gives

$$
\mathbb{E}_{\tilde{w}}\left[r_{T}\right] \geq c_{5} \sigma \delta^{2} \sqrt{\frac{1}{T \ln T}} .
$$

Upon averaging over all BM realizations, this implies

$$
\begin{aligned}
& \mathbb{E}\left[r_{T}\right]= \mathbb{E}\left[r_{T} \mid \tilde{W} \in \Pi_{T}\right] \operatorname{Pr}\left[\tilde{W} \in \Pi_{T}\right] \\
&+\mathbb{E}\left[r_{T} \mid \tilde{W} \in \Pi_{T}^{c}\right] \operatorname{Pr}\left[\tilde{W} \in \Pi_{T}^{c}\right] \\
& \geq \mathbb{E}\left[r_{T} \mid \tilde{W} \in \Pi_{T}\right] \operatorname{Pr}\left[\tilde{W} \in \Pi_{T}\right] \\
& \geq c_{5} \sigma \delta^{2} \sqrt{\frac{1}{T \ln T}} \mathbb{P}[\mathcal{T}]
\end{aligned}
$$




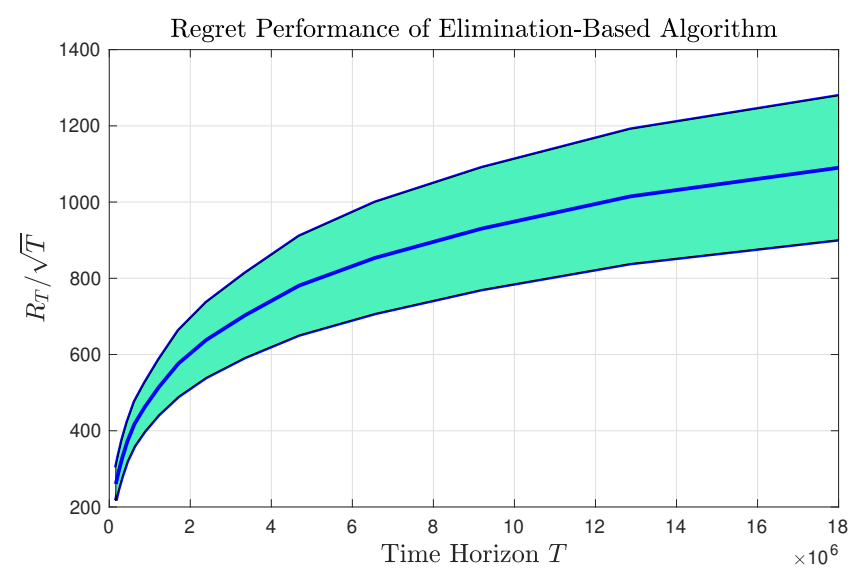

Fig. 3: Plot of $R_{T} / \sqrt{T}$ against $T$.

$$
=\Omega\left(\sigma \sqrt{\frac{1}{T \log T}}\right),
$$

where in 770 we define $\Pi_{\mathcal{T}}$ to be the set of sample paths of the BM $W$ such that the induced $x_{M}, r^{+}(\cdot)$ and $r^{-}(\cdot)$ satisfy the events defining $\mathcal{T}$ in Definition 3 , (72) follows from (69), and (73) follows from Lemma 8 with $\delta$ chosen to a constant value (e.g., $\delta=0.5$ ). This yields the desired lower bound for the simple regret.

As shown in (48), for achievability results it is trivial to obtain $\mathbb{E}\left[r_{T}\right] \leq \mathbb{E}\left[R_{T}\right] / T$. The contrapositive statement is that for converse results, a universal lower bound of on $\mathbb{E}\left[r_{T}\right]$ implies the same universal lower bound for $\mathbb{E}\left[R_{T}\right] / T$, and it follows that $\mathbb{E}\left[R_{T}\right]=\Omega\left(\sigma \sqrt{\frac{T}{\log T}}\right)$, as desired.

\section{EXPERIMENTS}

Theorems 1 and 2 state that the expected cumulative regret achieved by Algorithm 1 is at most $\mathcal{O}\left(\sigma T^{\frac{1}{2}} \log T\right)$ and at least $\Omega\left(\sigma T^{\frac{1}{2}} / \sqrt{\log T}\right)$, so the growth rate is roughly $\sqrt{T}$. We corroborate these theoretical findings using numerical experiments. We implement and run Algorithm 1, varying the time horizon $T$ from $10^{5}$ and $1.8 \times 10^{7}$. The noise variance is set to $\sigma^{2}=0.25$. We generate 50 independent realizations of a $\mathrm{BM}$ on $[0,1]$. For each realization, we run the algorithm 100 times, each time corresponding to different realizations of the noise. We calculate the mean and standard deviation of the cumulative regret (over all 5000 runs) at each $T$, and plot the average $R_{T} / \sqrt{T}$ against $T$. These are shown in Figure 3 , error bars indicate one standard deviation from the mean.

Figure 3 shows that as $T \rightarrow \infty, R_{T} / \sqrt{T}$ appears to be bounded, or at least growing very slowly (e.g., due to logarithmic factors), as $T$ increases. This indicates that $R_{T}$ scales roughly as $\sqrt{T}$, corroborating our theoretical findings.

\section{CONCLUSION}

We have established upper and lower bounds on the smallest possible simple regret and cumulative regret for the noisy optimization of a Brownian motion. These bounds are tight up to a logarithmic factor in $T$. Our results complement the existing bounds on Bayesian optimization with smooth functions, revealing that in fact the cumulative regret enjoys similar scaling laws in the smooth and non-smooth scenarios.
In future work, it would be interesting to determine whether similar results hold for other non-smooth processes, such as the Ornstein-Uhlenbeck (OU) process or multi-dimensional variants of BM. We expect that some of these (e.g. OU process with known parameters) should be possible with similar techniques to ours, by leveraging the solution of its accompanying stochastic differential equation. However, we also expect other stochastic processes is more challenging (e.g. processes with unknown parameters involving stochastic filtering) and possibly require new confidence bounds, Höldertype continuity results, and so on. We leave such investigations to future work.

\section{Proofs of Auxiliary Lemmas}

\section{A. Proof for Lemma 2 (Probability of Event $\mathcal{M}$ )}

We start with the proxy-Lipschitz condition $\mathcal{C}$ of Grill et al. [4], in which we use $\delta$ as the argument for the probability of "failure", recall that the event $\mathcal{C}$ is defined as follows:

$$
\mathcal{C} \triangleq \bigcap_{h=0}^{\infty} \bigcap_{k=0}^{2^{h}-1}\left\{\max _{x \in\left[\frac{k}{2^{h}}, \frac{k+1}{2^{h}}\right]} W_{x} \leq B\left(\left[\frac{k}{2^{h}}, \frac{k+1}{2^{h}}\right]\right)\right\},
$$

where $B([a, b])=\max \left(W_{a}, W_{b}\right)+\eta_{\delta}(b-a)$, and $\eta_{\delta}(\cdot)$ is defined in $(10)$.

According to [4, Lemma 2], $\mathcal{C}$ occurs with probability at least $1-\delta^{5}$. We can also define an analogous event $\mathcal{C}^{\prime}$ (involving lower bounds instead of upper bounds), which similarly holds with probability at least $1-\delta^{5}$, and is defined as follows:

$$
\mathcal{C}^{\prime} \triangleq \bigcap_{h=0}^{\infty} \bigcap_{k=0}^{2^{h}-1}\left\{\min _{x \in\left[\frac{k}{2^{h}}, \frac{k+1}{2^{h}}\right]} W_{x} \geq B^{\prime}\left(\left[\frac{k}{2^{h}}, \frac{k+1}{2^{h}}\right]\right)\right\},
$$

where $B^{\prime}([a, b])=\min \left(W_{a}, W_{b}\right)-\eta_{\delta}(b-a)$.

We then define two sets of sub-events to prove that $\mathcal{M}_{1}$ and $\mathcal{M}_{2}$ hold with high probability: For each $h \geq 1$ and $k \in\left\{0,1, \ldots, 2^{h}-1\right\}$, define

$$
\mathcal{G}_{h, k} \triangleq\left\{\left|W_{\frac{k+1}{2^{h}}}-W_{\frac{k}{2^{h}}}\right|>\alpha_{\delta}\left(2^{-h}\right)\right\},
$$

and for each $h \geq 1$ and $k \in \mathcal{J}_{h}$, define

$$
\mathcal{H}_{h, k} \triangleq\left\{\left|\bar{y}_{\frac{k+1}{2^{h}}}^{(h)}-W_{\frac{k+1}{2^{h}}}\right|>\alpha_{\delta}\left(2^{-h}\right)\right\},
$$

where $\alpha_{\delta}(\cdot)$ is defined in $(10)$, and we recall that $\bar{y}_{x}^{(h)}=$ Average $\left(\left\{y_{s} \mid 1 \leq s \leq T_{h}, x_{s}=x\right\}\right)$.

Since for any $k \in\left\{0,1, \ldots, 2^{h}-1\right\}$, the differences $W_{\frac{k+1}{2^{h}}}-$ $W_{\frac{k}{2^{h}}}$ are normally distributed with variance $2^{-h}$, we can upper bound them by

$$
\alpha_{\delta}\left(2^{-h}\right)=\sqrt{2^{-h} \ln \left(\frac{1}{2 \pi\left(2^{-h} \delta\right)^{6}}\right)}
$$

with probability at least $1-2^{-2 h} \delta^{3}$ according to Lemma 1 This implies that each $\mathcal{G}_{h, k}$ holds with probability at most $2^{-2 h} \delta^{3}$. Since at least $n_{h}=\sigma^{2} 2^{h}$ samples have been taken at each point in $\mathcal{J}_{h}$ (see Algorithm 11), the variance of the 
difference between the actual value and the average over noisy samples at these points is reduced to at most $2^{-h}$, and each $\mathcal{H}_{h, k}$ holds with probability at most $2^{-2 h} \delta^{3}$ similarly to the argument for $\mathcal{G}_{h, k}$.

By the union bound on all the sub-events, and summing the probabilities with a geometric series, we conclude that $\mathbb{P}\left[\mathcal{M}_{1}\right] \geq 1-\delta^{3}$ and $\mathbb{P}\left[\mathcal{M}_{2}\right] \geq 1-\delta^{3}$. Note that $\mathcal{M}_{2} \cap \mathcal{C} \cap \mathcal{C}^{\prime}$ implies $\mathcal{M}_{3}$ and $\mathcal{M}_{4}$. Hence, the intersection of $\mathcal{M}_{1}, \mathcal{M}_{2}$, $\mathcal{C}$ and $\mathcal{C}^{\prime}$ implies $\mathcal{M}$, and by the union bound, $\mathbb{P}[\mathcal{M}] \geq 1-$ $2 \delta^{3}-2 \delta^{5} \geq 1-\delta^{2}$.

\section{B. Proof of Equation (46) (Expected Regret Given $\mathcal{M}^{c}$ )}

Here we show that $\mathbb{E}\left[R_{T} \mid \mathcal{A}\right]=\mathcal{O}\left(T \sqrt{\log \frac{1}{\mathbb{P}[\mathcal{A}]}}\right)$ for any event $\mathcal{A}$. For brevity, we define $\delta_{0}=\mathbb{P}[\mathcal{A}]$.

We first note from Lemma A.1 in Appendix A (in the supplementary material) that the maximum (and similarly, the minimum) of a Brownian motion has the same distribution as the absolute value of an $\mathcal{N}(0,1)$ random variable. By Lemma 1 it follows that (unconditionally) $\max _{x \in[0,1]} W_{x}-$ $\min _{x \in[0,1]} W_{x} \leq c \sqrt{\log \frac{1}{\delta}}$ with probability at least $1-\delta^{3}$, where $\delta$ is arbitrary and $c$ is an absolute constant. Note that the cumulative regret up to time $T$ is trivially upper bounded by $T$ times Gap $:=\max _{x \in[0,1]} W_{x}-\min _{x \in[0,1]} W_{x}$.

To move to the case with conditioning on $\mathcal{A}$, we write

$$
\begin{aligned}
& \mathbb{E}[\operatorname{Gap} \mid \mathcal{A}] \\
& =\mathbb{E}\left[\operatorname{Gap} \mathbf{1}\left\{\operatorname{Gap} \leq c \sqrt{\log \frac{1}{\delta_{0}}}\right\} \mid \mathcal{A}\right] \\
& \quad+\mathbb{E}\left[\operatorname{Gap} \mathbf{1}\left\{\operatorname{Gap}>c \sqrt{\log \frac{1}{\delta_{0}}}\right\} \mid \mathcal{A}\right] \\
& \leq c \sqrt{\log \frac{1}{\delta_{0}}}+\frac{1}{\delta_{0}} \mathbb{E}\left[\operatorname{Gap} \mathbf{1}\left\{\operatorname{Gap}>c \sqrt{\log \frac{1}{\delta_{0}}}\right\}\right],
\end{aligned}
$$

where the second term of $(80)$ uses $\mathbb{E}[Z \mid \mathcal{A}]=\frac{\mathbb{E}[Z 1\{\mathcal{A}\}]}{\mathbb{P}[\mathcal{A}]} \leq$ $\frac{\mathbb{E}[Z]}{\mathbb{P}[\mathcal{A}]}$ for any non-negative random variable $Z$ and event $\mathcal{A}$.

Using the formula $\mathbb{E}[Z]=\int_{0}^{\infty} \mathbb{P}[Z \geq z] \mathrm{d} z$ for a nonnegative random variable $Z$, and using the above arguments for (unconditionally) bounding Gap with high probability, it is straightforward to establish that

$$
\mathbb{E}\left[\operatorname{Gap} \mathbf{1}\left\{\operatorname{Gap}>c \sqrt{\log \frac{1}{\delta_{0}}}\right\}\right]=\mathcal{O}\left(\delta_{0}^{3} \log \frac{1}{\delta_{0}}\right) .
$$

As a result, for any $\delta_{0}$ bounded away from one, the first term has the dominant scaling behavior in 80 , and we obtain $\mathbb{E}[$ Gap $\mid \mathcal{A}]=\mathcal{O}\left(\sqrt{\log \frac{1}{\delta_{0}}}\right)$ and hence $\mathbb{E}\left[R_{T} \mid \mathcal{A}\right]=$ $\mathcal{O}\left(T \sqrt{\log \frac{1}{\delta_{0}}}\right)$.

\section{Proof of Lemma 6 (Running Maximum Lower Bound for a Brownian Meander)}

According to [25], the distribution function of the running maximum conditioned on the running minimum being positive can be expressed as follows:

$$
\mathbb{P}\left[\max _{0 \leq z \leq s} W_{z}<x \mid \min _{0<z \leq t} W_{z}>0, W_{0}=0\right]
$$

$$
=\int_{0}^{x} H(y) \frac{\int_{0}^{\infty}\left[\exp \left(-\frac{(w-y)^{2}}{2(t-s)}\right)-\exp \left(-\frac{(w+y)^{2}}{2(t-s)}\right) \frac{\mathrm{d} w}{\sqrt{2 \pi(t-s)}}\right]}{\int_{0}^{\infty} \frac{w}{t \sqrt{2 \pi t}} \exp \left(-\frac{w^{2}}{2 t}\right) \mathrm{d} w} \mathrm{~d} y,
$$

where

$$
H(y) \triangleq \sum_{k=-\infty}^{\infty} \frac{y-2 k x}{s \sqrt{2 \pi s}} \exp \left(-\frac{(y-2 k x)^{2}}{2 s}\right) .
$$

We first upper bound $H(y)$ in terms of $a=$ $\max \left(0,\left\lfloor\frac{\sqrt{s}-x}{2 x}\right\rfloor\right)$ with $\left.v(z) \triangleq z \exp \left(-\frac{z^{2}}{2}\right):\right\rfloor^{7}$

$$
\begin{aligned}
H(y)= & \sum_{k=-\infty}^{\infty}\left[\frac{y-2 k x}{s \sqrt{2 \pi s}} \exp \left(-\frac{(y-2 k x)^{2}}{2 s}\right)\right] \\
= & \frac{y}{s \sqrt{2 \pi s}} \exp \left(-\frac{y^{2}}{2 s}\right) \\
& +\frac{1}{s \sqrt{2 \pi}} \sum_{k=1}^{\infty}\left[v\left(\frac{2 k x+y}{\sqrt{s}}\right)-v\left(\frac{2 k x-y}{\sqrt{s}}\right)\right] \\
\leq & \frac{y}{s \sqrt{2 \pi s}} \exp \left(-\frac{y^{2}}{2 s}\right) \\
& +\frac{1}{s \sqrt{2 \pi}} \sum_{k=1}^{a}\left[v\left(\frac{2 k x+y}{\sqrt{s}}\right)-v\left(\frac{2 k x-y}{\sqrt{s}}\right)\right] \\
\leq & \frac{y}{s \sqrt{2 \pi s}} \exp \left(-\frac{y^{2}}{2 s}\right)+\frac{1}{s \sqrt{2 \pi}} \sum_{k=1}^{a} \frac{2 y}{\sqrt{s}} \\
\leq & \frac{y}{s \sqrt{2 \pi s}} \exp \left(-\frac{y^{2}}{2 s}\right)+\frac{1}{s \sqrt{2 \pi}}\left(\frac{\sqrt{s}}{2 x}\right) \frac{2 y}{\sqrt{s}} \\
\leq & \frac{y}{s \sqrt{2 \pi s}} \exp \left(-\frac{y^{2}}{2 s}\right)+\frac{y}{x s \sqrt{2 \pi}},
\end{aligned}
$$

where 86 follows from the monotonically decreasing property of the function $v(z)=z \exp \left(-\frac{z^{2}}{2}\right)$ for $z>1$ (since $x \geq y$, we have $\frac{2 k x+y}{\sqrt{s}}>\frac{2 k x-y}{\sqrt{s}} \geq \frac{\sqrt{s}+x-y}{\sqrt{s}} \geq 1$ for $k \geq a+1=\left\lfloor\frac{\sqrt{s}-x}{2 x}\right\rfloor+1 \geq\left\lceil\frac{\sqrt{s}+x}{2 x}\right\rceil$ ), (87ך) follows from the fact that the function $v(z)=z \exp \left(-\frac{z^{2}}{2}\right)$ has Lipschitz constant 1 on the domain $(0,1)$, and 89 follows from $a \leq \frac{\sqrt{s}}{2 x}$.

The distribution function can then be upper bounded as follows:

$$
\begin{aligned}
& \mathbb{P}\left[\max _{0 \leq z \leq s} W_{z}<x \mid \min _{0<z \leq t} W_{z}>0, W_{0}=0\right] \\
& =\int_{0}^{x} H(y) \frac{\int_{0}^{\infty}\left[\exp \left(-\frac{(w-y)^{2}}{2(t-s)}\right)-\exp \left(-\frac{(w+y)^{2}}{2(t-s)}\right)\right] \frac{\mathrm{d} w}{\sqrt{2 \pi(t-s)}}}{\int_{0}^{\infty} \frac{w}{t \sqrt{2 \pi t}} \exp \left(-\frac{w^{2}}{2 t}\right) \mathrm{d} w} \mathrm{~d} y
\end{aligned}
$$

$$
=\int_{0}^{x} H(y) \frac{\int_{-\frac{y}{\sqrt{t-s}}}^{\infty} \exp \left(-\frac{z^{2}}{2}\right) \frac{\mathrm{d} z}{\sqrt{2 \pi}}-\int_{\frac{y}{\sqrt{t-s}}}^{\infty} \exp \left(-\frac{z^{2}}{2}\right) \frac{\mathrm{d} z}{\sqrt{2 \pi}}}{\int_{0}^{\infty} \frac{w}{t \sqrt{2 \pi t}} \exp \left(-\frac{w^{2}}{2 t}\right) \mathrm{d} w} \mathrm{~d} y
$$$$
=\int_{0}^{x} H(y) \frac{\int_{-\frac{y}{\sqrt{t-s}}}^{\frac{y}{\sqrt{t-s}}} \exp \left(-\frac{z^{2}}{2}\right) \frac{\mathrm{d} z}{\sqrt{2 \pi}}}{\int_{0}^{\infty} \frac{z}{\sqrt{2 \pi t}} \exp \left(-\frac{z^{2}}{2}\right) \mathrm{d} w} \mathrm{~d} y
$$ 
$\leq \int_{0}^{x}\left[\frac{y}{s \sqrt{2 \pi s}} \exp \left(-\frac{y^{2}}{2 s}\right)+\frac{y}{x s \sqrt{2 \pi}}\right] \frac{\int_{-\frac{y}{\sqrt{t-s}}}^{\frac{y}{\sqrt{t-s}}} \exp \left(-\frac{z^{2}}{2}\right) \frac{\mathrm{d} z}{\sqrt{2 \pi}}}{\frac{1}{\sqrt{2 \pi t}}} \mathrm{~d} y$

where (91) follows from the change of variables $z=w-$ $y, z=w+y$, and 93 follows from (89) and a direct evaluation of the integral in the denominator.

We now consider two cases separately. First, if $t>2 s$ :

$$
\begin{aligned}
& \mathbb{P}\left[\max _{0 \leq z \leq s} W_{z}<x \mid \min _{0<z \leq t} W_{z}>0, W_{0}=0\right] \\
& \leq \int_{0}^{x}\left[\frac{y}{s \sqrt{2 \pi s}} \exp \left(-\frac{y^{2}}{2 s}\right)+\frac{y}{x s \sqrt{2 \pi}}\right] \frac{2 \frac{1}{\sqrt{2 \pi}} \frac{y}{\sqrt{t-s}}}{\frac{1}{\sqrt{2 \pi t}}} \mathrm{~d} y \\
& =\frac{2 \sqrt{t}}{\sqrt{2 \pi s(t-s)}} \int_{0}^{x}\left(\frac{y^{2}}{s} \exp \left(-\frac{y^{2}}{2 s}\right)+\frac{y^{2}}{x \sqrt{s}}\right) \mathrm{d} y \\
& =\frac{2 \sqrt{t}}{\sqrt{2 \pi s(t-s)}}\left[\sqrt{s} \int_{0}^{\frac{x}{\sqrt{s}}} \exp \left(-\frac{z^{2}}{2}\right) \mathrm{d} z\right. \\
& \leq \frac{2 x \sqrt{t}}{\sqrt{2 \pi s(t-s)}}\left[1-\exp \left(-\frac{x^{2}}{2 s}\right)+\frac{x}{3 \sqrt{s}}\right] \\
& \leq \frac{1}{\sqrt{2 \pi}} \sqrt{\frac{t}{t-s}}\left(\frac{x}{\sqrt{s}}\right)^{3}+\sqrt{\frac{t}{t-s}} \frac{x^{2} \sqrt{2}}{3 \sqrt{\pi} s} \\
& <\frac{1}{\sqrt{\pi}}\left(\frac{x}{\sqrt{s}}\right)^{3}+\frac{2}{3 \sqrt{\pi}}\left(\frac{x}{\sqrt{s}}\right)^{2} \\
& \leq \frac{5}{6 \sqrt{\pi}}\left(\frac{x}{\sqrt{s}}\right)^{2} \\
& \leq \frac{1}{2}\left(\frac{x}{\sqrt{s}}\right)^{2}
\end{aligned}
$$

where (94) follows by upper bounding the exponential function $\exp \left(-\frac{z^{2}}{2}\right)$ by 1 in the inner integral of 93, 96 follows from integration by parts, 97 follows by upper bounding the exponential function $\exp \left(-\frac{z^{2}}{2}\right)$ by 1 , 98) follows from the fact that $1-\frac{x^{2}}{2 s} \leq \exp \left(-\frac{x^{2}}{2 s}\right)$ as $x^{2}<\frac{1}{4} s<2 s$, and 99) follows from $\frac{t}{t-s}<2$ (since $t>2 s$ ) and $x<\sqrt{s} / 2$ (assumed in the lemma).

As for the other case, if $t \leq 2 s$, then:

$$
\begin{aligned}
& \mathbb{P}\left[\max _{0<z \leq s} W_{z}<x \mid \min _{0 \leq z \leq t} W_{z}>0, W_{0}=0\right] \\
& \leq \int_{0}^{x}\left[\frac{y}{s \sqrt{2 \pi s}} \exp \left(-\frac{y^{2}}{2 s}\right)+\frac{y}{x s \sqrt{2 \pi}}\right] \frac{1}{\frac{1}{\sqrt{2 \pi t}}} \mathrm{~d} y \\
& =\sqrt{t} \int_{0}^{x}\left[\frac{y}{s \sqrt{s}} \exp \left(-\frac{y^{2}}{2 s}\right)+\frac{y}{x s}\right] \mathrm{d} y \\
& =\sqrt{t} \int_{0}^{x / \sqrt{s}} \frac{z}{\sqrt{s}} \exp \left(-\frac{z^{2}}{2}\right) \mathrm{d} z+\sqrt{t}\left[\frac{y^{2}}{2 x s}\right]_{y=0}^{y=x} \\
& =\frac{\sqrt{t}}{\sqrt{s}}\left[-\exp \left(-\frac{z^{2}}{2}\right)\right]_{z=0}^{z=\frac{x}{\sqrt{s}}}+\frac{x \sqrt{t}}{2 s} \\
& =\frac{\sqrt{t}}{\sqrt{s}}\left[1-\exp \left(-\frac{x^{2}}{2 s}\right)\right]+\frac{x \sqrt{t}}{2 s}
\end{aligned}
$$

$$
\begin{aligned}
& \leq \frac{x^{2} \sqrt{t}}{2 s \sqrt{s}}+\frac{x \sqrt{t}}{2 s} \\
& \leq \frac{1}{\sqrt{2}}\left(\frac{x}{\sqrt{s}}\right)^{2}+\frac{x}{\sqrt{2 s}} \\
& \leq \sqrt{2} \frac{x}{\sqrt{s}},
\end{aligned}
$$

where (102) follows from the fact that the numerator in 93 $\int_{-\frac{y}{\sqrt{t-s}}}^{\frac{y}{\sqrt{t-s}}} \exp \left(-\frac{z^{2}}{2}\right) \frac{\mathrm{d} z}{\sqrt{2 \pi}}=\mathbb{P}\left[-\frac{y}{\sqrt{t-s}} \leq Z \leq \frac{y}{\sqrt{t-s}}\right] \leq 1$ for $Z \stackrel{\mathcal{N}}{\sim}(0,1)$, 104 applies the change of variable $z=\frac{y}{\sqrt{s}}$, 107) follows from the fact that $\exp (-x)>1-x$ for all $x>0$, and (108) follows from $t \leq 2 s$ and $x<\frac{1}{2} \sqrt{s} \leq \sqrt{s}$ (assumed in the lemma). Hence, the probability of the complement event is lower bounded as follows:

$$
\begin{aligned}
& \mathbb{P}\left[\max _{0<z \leq s} W_{z} \geq x \mid \min _{0 \leq z \leq t} W_{z}>0, W_{0}=0\right] \\
& \quad \geq \begin{cases}1-\frac{1}{2}\left(\frac{x}{\sqrt{s}}\right)^{2} & \text { if } t>2 s \\
1-\sqrt{2} \frac{x}{\sqrt{s}} & \text { if } t \leq 2 s .\end{cases}
\end{aligned}
$$

\section{Proof of Lemma 7 (Running Minimum Lower Bound for a} Brownian Meander)

We have

$$
\begin{aligned}
& \mathbb{P}\left[\min _{0 \leq z \leq t} W_{z}>\varepsilon \mid \min _{0 \leq z \leq t} W_{z}>0, W_{0}=u\right] \\
& =\frac{\mathbb{P}\left[\min _{0 \leq z \leq t} W_{z}>\varepsilon, \min _{0 \leq z \leq t} W_{z}>0 \mid W_{0}=u\right]}{\mathbb{P}\left[\min _{0 \leq z \leq t} W_{z}>0 \mid W_{0}=u\right]} \\
& =\frac{\mathbb{P}\left[\min _{0 \leq z \leq t} W_{z}>\varepsilon \mid W_{0}=u\right]}{\mathbb{P}\left[\min _{0 \leq z \leq t} W_{z}>0 \mid W_{0}=u\right]} \\
& =\frac{\int_{\varepsilon}^{\infty}\left[\exp \left(-\frac{(y-u)^{2}}{2 t}\right)-\exp \left(-\frac{(y+u-2 \varepsilon)^{2}}{2 t}\right)\right] \mathrm{d} y}{\int_{0}^{\infty}\left[\exp \left(-\frac{(y-u)^{2}}{2 t}\right)-\exp \left(-\frac{(y+u)^{2}}{2 t}\right)\right] \mathrm{d} y} \\
& =\frac{\frac{1}{\sqrt{2 \pi}} \int_{0}^{\frac{u-\varepsilon}{\sqrt{t}}} \exp \left(-\frac{1}{2} z^{2}\right) \mathrm{d} z}{\frac{1}{\sqrt{2 \pi}} \int_{0}^{\frac{u}{\sqrt{t}}} \exp \left(-\frac{1}{2} z^{2}\right) \mathrm{d} z} \\
& \geq \frac{\frac{u-\varepsilon}{\sqrt{t}}}{\frac{u}{\sqrt{t}}}=\frac{u-\varepsilon}{u},
\end{aligned}
$$

where (113) follows from the joint distribution of Brownian meander (with initial value $u$ ) and its running minimum as stated in Lemma A.3 in Appendix A. (114) follows similar steps to 900-92) (recall also that we assumed $0<\epsilon<u$ ), and 115) follows from the fact that $e^{-z^{2} / 2}$ is monotonically decreasing on the positive real line.

\section{E. Proof of Lemma 8 (Probability of Event $\mathcal{T}$ )}

We lower bound the probability of each $\mathcal{T}_{i}$ separately for $i=1,2,3$.

Bounding $\mathbb{P}\left[\mathcal{T}_{1}\right]$. Let $\delta_{1}=\Delta^{\eta}$ denote the target error probability of event $\mathcal{T}_{1}$. We first define three running maxima 
of three regions as follows:

$$
\begin{aligned}
& M_{1}=\max _{x \in[-\Delta, 2 \Delta]} \tilde{W}_{x}, \quad M_{2}=\max _{x \in[2 \Delta, 1-2 \Delta]} \tilde{W}_{x}, \\
& M_{3}=\max _{x \in[1-2 \Delta, 1+\Delta]} \tilde{W}_{x} .
\end{aligned}
$$

We consider two separate events:

$$
E_{1}=\left\{M_{1}<M_{2}\right\}, \text { and } E_{2}=\left\{\max \left(M_{1}, M_{2}\right)>M_{3}\right\},
$$

whose intersection directly implies $\mathcal{T}_{1}$. To simplify notation, we define $Z$ to be a standard normal random variable independent of the other defined random variables.

To bound the probability of $E_{1}$, we first establish a highprobability upper bound $\hat{M}_{1}=\sqrt{2 \Delta \ln \frac{8}{\delta_{1} \sqrt{2 \pi}}}$ for $M_{1}$ :

$$
\begin{aligned}
\mathbb{P}\left[M_{1} \leq \hat{M}_{1}\right] & =\mathbb{P}\left[\left|\tilde{W}_{2 \Delta}\right| \leq \sqrt{2 \Delta \ln \frac{8}{\delta_{1} \sqrt{2 \pi}}}\right] \\
& =\mathbb{P}\left[|Z| \leq \sqrt{\ln \frac{8}{\delta_{1} \sqrt{2 \pi}}}\right] \\
& =1-2 Q\left(\sqrt{\ln \frac{8}{\delta_{1} \sqrt{2 \pi}}}\right) \\
& \geq 1-\frac{\delta_{1}}{4}
\end{aligned}
$$

where (118) follows from the distribution function of the running maximum of a BM (see Lemma A.1 in Appendix A), and (120) follows from Lemma 1 .

The lower bound on $M_{2}$ requires a two-part argument. First, we lower bound the value of $\tilde{W}_{2 \Delta}$ by $\hat{w}_{2 \Delta}=$ $-\sqrt{2 \Delta \ln \frac{8}{\delta_{1} \sqrt{2 \pi}}}:$

$$
\begin{aligned}
\mathbb{P}\left[\tilde{W}_{2 \Delta} \geq \hat{w}_{2 \Delta}\right] & =\mathbb{P}\left[Z \geq-\sqrt{\ln \frac{8}{\delta_{1} \sqrt{2 \pi}}}\right] \\
& =1-Q\left(\sqrt{\ln \frac{8}{\delta_{1} \sqrt{2 \pi}}}\right) \\
& \geq 1-\frac{\delta_{1}}{8} .
\end{aligned}
$$

Second, we lower bound the value of $M_{2}-\tilde{W}_{2 \Delta}$ by

$$
\hat{S}_{2}=\frac{\delta_{1} \sqrt{\pi}}{8 \sqrt{2}} \sqrt{1-4 \Delta} .
$$

To see this, we similarly use the distribution of running maximum of Brownian motion (Lemma A.1) to obtain

$$
\begin{aligned}
& \mathbb{P}\left[M_{2}-\tilde{W}_{2 \Delta} \geq \frac{\delta_{1} \sqrt{\pi}}{8 \sqrt{2}} \sqrt{1-4 \Delta}\right] \\
& =\mathbb{P}\left[|Z| \geq \frac{\delta_{1} \sqrt{\pi}}{8 \sqrt{2}}\right]=1-\mathbb{P}\left[|Z|<\frac{\delta_{1} \sqrt{\pi}}{8 \sqrt{2}}\right] \\
& =1-2 \int_{0}^{\frac{\delta_{1} \sqrt{\pi}}{8 \sqrt{2}}} \frac{1}{\sqrt{2 \pi}} \exp \left(-\frac{z^{2}}{2}\right) \mathrm{d} z \\
& \geq 1-2 \frac{\delta_{1} \sqrt{\pi}}{8 \sqrt{2}} \frac{1}{\sqrt{2 \pi}}=1-\frac{\delta_{1}}{8} .
\end{aligned}
$$

Hence, defining $\hat{M}_{2}=\hat{w}_{2 \Delta}+\hat{S}_{2}$ and applying the union bound, we obtain

$$
\begin{aligned}
\mathbb{P}\left[M_{2} \geq \hat{M}_{2}\right]= & \mathbb{P}\left[M_{2} \geq \hat{w}_{2 \Delta}+\hat{S}_{2}\right] \\
\geq 1- & {\left[1-\mathbb{P}\left[\tilde{W}_{2 \Delta} \geq \hat{w}_{2 \Delta}\right]\right] } \\
& \quad-\left[1-\mathbb{P}\left[M_{2}-\tilde{W}_{2 \Delta} \geq \hat{S}_{2}\right]\right] \\
\geq & 1-\frac{\delta_{1}}{4} .
\end{aligned}
$$

As we take $\delta_{1}=\Delta^{\eta}$ for some $\eta<\frac{1}{2}$, we have $\hat{M}_{2} \geq \hat{M}_{1}$ for sufficiently small $\Delta$, since $\hat{M}_{1}=\Theta(\sqrt{\Delta \log (1 / \Delta)})$ and $\hat{M}_{2}=\Theta\left(\Delta^{\eta}-\sqrt{\Delta \log (1 / \Delta)}\right)=\Theta\left(\Delta^{\eta}\right)$ as $\Delta \rightarrow 0$. Therefore,

$$
\begin{aligned}
& \mathbb{P}\left[M_{2} \geq M_{1}\right] \\
& \geq 1-\left[1-\mathbb{P}\left[M_{2} \geq \hat{M}_{2}\right]\right]-\left[1-\mathbb{P}\left[M_{1} \leq \hat{M}_{1}\right]\right] \\
& \geq 1-\frac{\delta_{1}}{2},
\end{aligned}
$$

following from (120) and (130) along with the union bound.

To bound the probability of $E_{2}$ (see (117)), we let $w=$ $\tilde{W}_{1-2 \Delta}$, and establish an upper bound on $M_{3}$ in terms of $w$ that holds with probability at least $1-\delta_{1} / 4$, namely $\hat{M}_{3}=w+$ $\sqrt{2 \Delta \ln \frac{8}{\delta_{1} \sqrt{2 \pi}}}$. This is proved similarly to 120 , so the details are omitted to avoid repetition. Similarly to (124), we define $\hat{w}_{1-2 \Delta}=\sqrt{(1-\Delta) \ln \frac{8}{\delta_{1} \sqrt{2 \pi}}}$ to be the high-probability upper bound of $\tilde{W}_{1-2 \Delta}$, namely, $\mathbb{P}\left[\tilde{W}_{1-2 \Delta} \leq \hat{w}_{1-2 \Delta}\right] \geq 1-\frac{\delta_{1}}{8}$.

Using the density function of $\tilde{W}_{1-2 \Delta}$, denoted by $f_{\tilde{W}_{1-2 \Delta}}(w)$, we lower bound the probability for $\max \left(M_{1}, M_{2}\right)$ to exceed $\hat{M}_{3}$ in (134)-(141) on the top of the next page, where

- 136) follows from the distribution function of the running maximum of Brownian bridge as stated (Lemma A.2 in the supplementary material, with $a=-\Delta, b=1-2 \Delta$, $w_{a}=0, w_{b}=w$, and $\left.x=\hat{M}_{3}\right)$ and the fact that the underlying probability is trivially one when the $\exp (-(\ldots))$ term is greater than one (since the running maximum is always at least as high as the two endpoints);

- 137) follows from the fact that $\exp (-a w+b)$ is decreasing in $w$ for any $a>0$ and $b$;

- (139) follows from the above-established fact that $W_{1-2 \Delta} \leq \hat{w}_{1-2 \Delta}$ with probability at least $1-\frac{\delta_{1}}{8}$, along with $\mathbb{P}[A] \exp (-\alpha)=\left(1-\mathbb{P}\left[A^{c}\right]\right) \exp (-\alpha) \geq$ $\exp (-\alpha)-\mathbb{P}\left[A^{c}\right]$ for $\alpha \geq 0$ (and hence $\exp (-\alpha) \leq 1$ );

- 140 follows from the fact that $\sqrt{1-\Delta}+\sqrt{2 \Delta}<2$ for $\Delta \in\left(0, \frac{1}{2}\right)$;

- the first inequality in (141) follows from the fact that $\exp (-x)>1$ for all $x>0$, along with the choice $\delta_{1}=$ $\Delta^{\eta}$ (when $\Delta$ is sufficiently small).

Hence, $E_{2}$ holds with probability at least $1-\delta_{1} / 2$. As the intersection of $E_{1}$ and $E_{2}$ implies $\mathcal{T}_{1}$, the maximum lies in between $2 \Delta$ and $1-2 \Delta$ with probability at least $1-\delta_{1}$ by the union bound on the two events.

Bounding $\mathbb{P}\left[\mathcal{T}_{2}\right]$. Recall from (54)-(56) that $M^{+}$and $x_{M}^{+}$ are respectively the maximum and maximizer of $W^{+}$. It will be useful to additionally define the following two points, where 


$$
\begin{aligned}
& \mathbb{P}\left[\max \left(M_{1}, M_{2}\right) \geq \hat{M}_{3}\right]=\mathbb{P}\left[\max _{x \in[-\Delta, 1-2 \Delta]} \tilde{W}_{x} \geq \hat{M}_{3}\right] \\
& =\int_{-\infty}^{\infty} \mathbb{P}\left[\max _{x \in[-\Delta, 1-2 \Delta]} \tilde{W}_{x} \geq \hat{M}_{3} \mid \tilde{W}_{1-2 \Delta}=w\right] f_{\tilde{W}_{1-2 \Delta}}(w) \mathrm{d} w \\
& =\int_{-\infty}^{\infty} \min \left(1, \exp \left[-\frac{2 \sqrt{2 \Delta \ln \frac{8}{\delta_{1} \sqrt{2 \pi}}}\left(w+\sqrt{2 \Delta \ln \frac{8}{\delta_{1} \sqrt{2 \pi}}}\right)}{1-\Delta}\right]\right) f_{\tilde{W}_{1-2 \Delta}}(w) \mathrm{d} w \\
& \geq \int_{-\infty}^{\hat{w}_{1-2 \Delta}} f_{\tilde{W}_{1-2 \Delta}}(w) \mathrm{d} w \exp \left[-\frac{2 \sqrt{2 \Delta \ln \frac{8}{\delta_{1} \sqrt{2 \pi}}}\left(\hat{w}_{1-2 \Delta}+\sqrt{2 \Delta \ln \frac{8}{\delta_{1} \sqrt{2 \pi}}}\right)}{1-\Delta}\right] \\
& =\mathbb{P}\left[\tilde{W}_{1-2 \Delta} \leq \hat{w}_{1-2 \Delta}\right] \exp \left[-\frac{2 \sqrt{2 \Delta \ln \frac{8}{\delta_{1} \sqrt{2 \pi}}}\left(\hat{w}_{1-2 \Delta}+\sqrt{2 \Delta \ln \frac{8}{\delta_{1} \sqrt{2 \pi}}}\right)}{1-\Delta}\right] \\
& \geq \exp \left[-\frac{2 \sqrt{2 \Delta \ln \frac{8}{\delta_{1} \sqrt{2 \pi}}}\left(\sqrt{(1-\Delta) \ln \frac{8}{\delta_{1} \sqrt{2 \pi}}}+\sqrt{2 \Delta \ln \frac{8}{\delta_{1} \sqrt{2 \pi}}}\right)}{1-\Delta}\right]-\frac{\delta_{1}}{8} \\
& \geq \exp \left(-4 \sqrt{2 \Delta} \ln \frac{8}{\delta_{1} \sqrt{2 \pi}}\right)-\frac{\delta_{1}}{8} \\
& >1-4 \sqrt{2 \Delta} \ln \frac{8}{\delta_{1} \sqrt{2 \pi}}-\frac{\delta_{1}}{8} \geq 1-\frac{\delta_{1}}{4},
\end{aligned}
$$

$\delta^{\prime} \triangleq 0.1 \delta$

$$
\begin{aligned}
& x_{M}^{+, \mathrm{L}} \triangleq \sup \left\{x \mid x<x_{M}^{+}, W_{x}^{+}=M^{+}-\delta^{\prime} \sqrt{2 \Delta}\right\} \\
& x_{M}^{+, \mathrm{R}} \triangleq \inf \left\{x \mid x>x_{M}^{+}, W_{x}^{+}=M^{+}-\delta^{\prime} \sqrt{2 \Delta}\right\} .
\end{aligned}
$$

We then consider the following auxiliary events:

$$
\begin{aligned}
& \mathcal{T}_{2}^{(\mathrm{A})} \triangleq\left\{\min _{x \in\left[x_{M}^{+}-\Delta, x_{M}^{+}\right]} W_{x}^{+} \leq M^{+}-\left(\delta^{\prime}\right)^{2} \sqrt{2 \Delta}\right\} \\
& \mathcal{T}_{2}^{(\mathrm{B})} \triangleq\left\{\min _{x \in\left[x_{M}^{+}, x_{M}^{+}+\Delta\right]} W_{x}^{+} \leq M^{+}-\left(\delta^{\prime}\right)^{2} \sqrt{2 \Delta}\right\} \\
& \mathcal{T}_{2}^{(\mathrm{C})} \triangleq\left\{\max _{x \in\left[0, x_{M}^{+, \mathrm{L}}\right]} W_{x}^{+} \leq M^{+}-\left(\delta^{\prime}\right)^{2} \sqrt{2 \Delta}\right\} \\
& \mathcal{T}_{2}^{(\mathrm{D})} \triangleq\left\{\max _{x \in\left[x_{M}^{+, \mathrm{R}}, 1\right]} W_{x}^{+} \leq M^{+}-\left(\delta^{\prime}\right)^{2} \sqrt{2 \Delta}\right\} .
\end{aligned}
$$

By definition, $\mathcal{T}_{2}^{(\mathrm{A})}$ is equivalent to $\left\{x_{M}^{+, \mathrm{L}} \geq x_{M}^{+}-\Delta\right\}$, and $\mathcal{T}_{2}^{(\mathrm{B})}$ is equivalent to $\left\{x_{M}^{+, \mathrm{R}} \leq x_{M}^{+}+\Delta\right\}$. The intersection of all four events and $\mathcal{T}_{1}$ implies $\mathcal{T}_{2}$, since if events $\mathcal{T}_{2}^{(\mathrm{A})}$ and $\mathcal{T}_{2}^{(\mathrm{B})}$ hold, then there is a point in the $\Delta$-neighborhood of $x_{M}^{+}$with value $W_{x}^{+}=M-\delta^{\prime} \sqrt{2 \Delta}$; moreover, if events $\mathcal{T}_{2}^{(\mathrm{C})}$ and $\mathcal{T}_{2}^{(\mathrm{D})}$ simultaneously hold, $W^{+}$does not fall below $M-\left(\delta^{\prime}\right)^{2} \sqrt{2 \Delta}$ outside this neighborhood. As long as event $\mathcal{T}_{1}$ holds, both maxima $x_{M}^{+}$and $x_{M}^{-}$are within $[0,1]$, and it is safe to conclude that if $r^{+}(x)$ is smaller than $\left(\delta^{\prime}\right)^{2} \sqrt{2 \Delta}, x$ must be in the $\Delta$-neighborhood near $x_{M}^{+}$, and we can conclude that $r^{-}(x)=M-W_{x}^{-}=M^{+}-\tilde{W}_{x-2 \Delta}=r^{+}(x-2 \Delta)$ is larger than $\left(\delta^{\prime}\right)^{2} \sqrt{2 \Delta}$.
We now define two processes, representing left and right Brownian meanders (i.e., BM conditioned on being positive):

$$
\begin{aligned}
B_{2}^{\mathrm{L}}(x) \triangleq \frac{M^{+}-W_{x_{M}^{+}-x \cdot x_{M}^{+}}^{+}}{\sqrt{x_{M}^{+}}}, \text {and } \\
B_{2}^{\mathrm{R}}(x) \triangleq \frac{M^{+}-W_{x_{M}^{+}+x \cdot\left(1-x_{M}^{+}\right)}^{+}}{\sqrt{1-x_{M}^{+}}} .
\end{aligned}
$$

Specifically, Lemma A.4 in Appendix A formally states that $B_{2}^{\mathrm{L}}$ and $B_{2}^{\mathrm{R}}$ are identically distributed Brownian meanders, and furthermore, that they are independent from each other and from $x_{M}^{+}$. See Figure 4 for an example.

For any fixed $\hat{x}_{M}^{+}$in the range $[\Delta, 1-3 \Delta]$, we define an intermediate quantity $\xi \triangleq \frac{\delta^{\prime} \sqrt{2 \Delta}}{\sqrt{\hat{x}_{M}^{+}}}$and note that the following event holds for $B_{2}^{\mathrm{L}}$ by construction:

$\mathcal{B}_{2}^{\mathrm{L}} \triangleq\left\{\min _{x \in[0,1]} B_{2}^{\mathrm{L}}(x)>0, B_{2}^{\mathrm{L}}(0)=0, B_{2}^{\mathrm{L}}\left(1-\frac{x_{M}^{+, \mathrm{L}}}{\hat{x}_{M}^{+}}\right)=\xi\right\}$.

Since the pair $\left(B_{2}^{\mathrm{L}}, B_{2}^{\mathrm{R}}\right)$ is independent of $x_{M}^{+}$, we can condition on $x_{M}^{+}=\hat{x}_{M}^{+}$in accordance with event $\mathcal{T}_{1}$ as follows:

$$
\begin{aligned}
& \mathbb{P}\left[\mathcal{T}_{2}^{(\mathrm{A})} \mid x_{M}^{+}=\hat{x}_{M}^{+}\right] \\
& \geq \mathbb{P}\left[\max _{x \in\left[0, \Delta / \hat{x}_{M}^{+}\right]} B_{2}^{\mathrm{L}}(x) \geq \xi \delta^{\prime} \mid \mathcal{B}_{2}^{\mathrm{L}}\right] \\
& \geq 1-\max \left(\frac{1}{2}\left(\frac{\xi \delta^{\prime}}{\xi / \delta^{\prime}}\right)^{2}, 2\left(\delta^{\prime}\right)^{2}\right)
\end{aligned}
$$



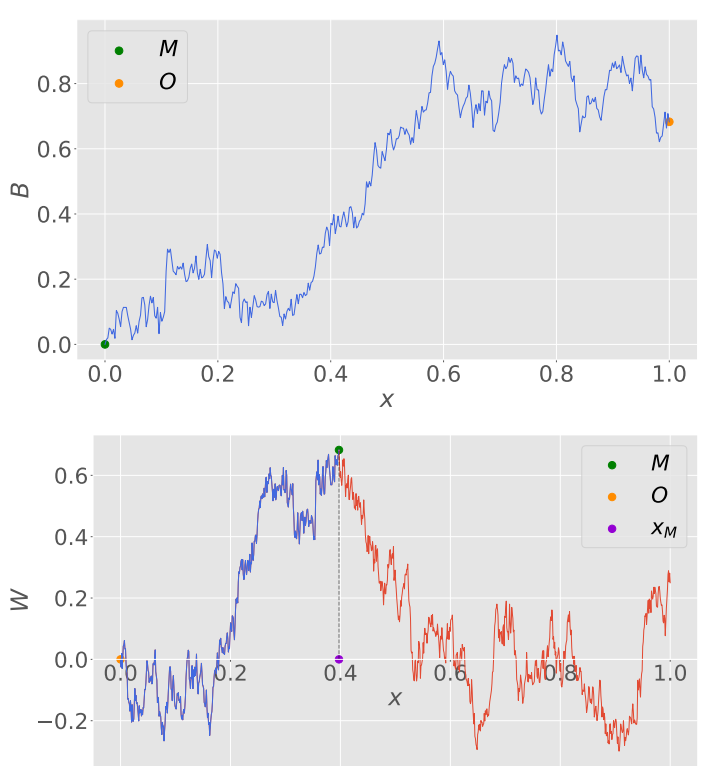

Fig. 4: Left Brownian meander example (top), and the original Brownian motion (bottom). Observe the correspondences between the points labeled $\mathrm{M}$ (maximum) and $\mathrm{O}$ (origin).

$$
=1-\max \left(\frac{1}{2}\left(\delta^{\prime}\right)^{4}, 2\left(\delta^{\prime}\right)^{2}\right)=1-2\left(\delta^{\prime}\right)^{2}
$$

where (151) follows from the definition of $B_{2}^{\mathrm{L}}$, and 152 follows from the minimum of two cases in Lemma 6 (with $x=\left(\delta^{\prime}\right)^{2} \sqrt{2 \Delta} / \sqrt{x_{M}^{+}}, s=\Delta / x_{M}^{+}$, and $\left.t=1\right){ }^{8}$

Averaging over $x_{M}^{+}$, we have

$$
\begin{aligned}
\mathbb{P}\left[\mathcal{T}_{2}^{(\mathrm{A})}\right] & =\int_{-\infty}^{\infty} f_{x_{M}^{+}}\left(\hat{x}_{M}^{+}\right) \mathbb{P}\left[\mathcal{T}_{2}^{(\mathrm{A})} \mid x_{M}^{+}=\hat{x}_{M}^{+}\right] \mathrm{d} \hat{x}_{M}^{+} \\
& =\int_{\Delta}^{1-3 \Delta} f_{x_{M}^{+}}\left(\hat{x}_{M}^{+}\right) \mathbb{P}\left[\mathcal{T}_{2}^{(\mathrm{A})} \mid x_{M}^{+}=\hat{x}_{M}^{+}\right] \mathrm{d} \hat{x}_{M}^{+} \\
& \geq \int_{\Delta}^{1-3 \Delta} f_{x_{M}^{+}}\left(\hat{x}_{M}^{+}\right)\left(1-\delta^{\prime} \sqrt{2}\right) \mathrm{d} \hat{x}_{M}^{+} \\
& =\left(1-2\left(\delta^{\prime}\right)^{2}\right) \mathbb{P}\left[x_{M}^{+} \in[\Delta, 1-3 \Delta]\right] \\
& \geq\left(1-2\left(\delta^{\prime}\right)^{2}\right)\left(1-\Delta^{\eta}\right) \\
& \geq 1-\Delta^{\eta}-2\left(\delta^{\prime}\right)^{2}
\end{aligned}
$$

where (156) follows from (152), and (158) follows from the fact that $\mathbb{P}\left[\mathcal{T}_{1}\right] \geq 1-\Delta^{\eta}$. Similarly, as $B_{2}^{\mathrm{L}}$ is equal in distribution to $B_{2}^{\mathrm{R}}, \mathbb{P}\left[\mathcal{T}_{2}^{(\mathrm{B})} \mid x_{M}^{-}=\hat{x}_{M}^{-}\right] \geq 1-2\left(\delta^{\prime}\right)^{2}$ (following from Lemma 6 for any $x_{M}^{-}$restricted in the range $[3 \Delta, 1-\Delta]$ in accordance with $\left.\mathcal{T}_{1}\right)$, and thus $\mathbb{P}\left[\mathcal{T}_{2}^{(\mathrm{B})}\right] \geq$ $\left(1-2\left(\delta^{\prime}\right)^{2}\right) \mathbb{P}\left[x_{M}^{-} \in[3 \Delta, 1-\Delta]\right] \geq\left(1-2\left(\delta^{\prime}\right)^{2}\right)\left(1-\Delta^{\eta}\right) \geq$ $1-\Delta^{\eta}-2\left(\delta^{\prime}\right)^{2}$.

Before considering $\mathcal{T}_{2}^{(\mathrm{C})}$, we use the Markov property to define a non-standard Brownian meander $\widetilde{B}_{2}^{\mathrm{L}}$ as follows: For

\footnotetext{
${ }^{8}$ Note that the Brownian meander in Lemma 6 does not contain any counterpart to the third condition in [150, but that condition is not playing a role here. It is simply an almost-sure event that holds by the definition of $x_{M}^{+, \mathrm{L}}$, so it makes no difference whether it is included in the conditioning or not. It is included in 150, for later convenience following 160 .
}

all $0 \leq x \leq \frac{x_{M}^{+, \mathrm{L}}}{\hat{x}_{M}^{+}}$

$$
\widetilde{B}_{2}^{\mathrm{L}}(x) \triangleq B_{2}^{\mathrm{L}}\left(x+1-\frac{x_{M}^{+, \mathrm{L}}}{\hat{x}_{M}^{+}}\right)
$$

The distribution of $\widetilde{B}_{2}^{\mathrm{L}}$ follows from $B_{2}^{\mathrm{L}}$ in the domain $[1-$ $\left.x_{M}^{+, \mathrm{L}} / \hat{x}_{M}^{+}, 1\right]$ to be a Brownian meander with starting value $\xi$, due to the Markov property of BM. The counterpart $\widetilde{\mathcal{B}}_{2}^{\mathrm{L}}$ to the event $\mathcal{B}_{2}^{\mathrm{L}}$ in 150 is as follows:

$$
\widetilde{\mathcal{B}}_{2}^{\mathrm{L}} \triangleq\left\{\min _{x \in\left[0, x_{M}^{+\mathrm{L}} / \hat{x}_{M}^{+}\right]} \widetilde{B}_{2}^{\mathrm{L}}(x)>0, \widetilde{B}_{2}^{\mathrm{L}}(0)=\xi\right\}
$$

Next, we lower bound $\mathbb{P}\left[\mathcal{T}_{2}^{(\mathrm{C})}\right]$ by conditioning on $x_{M}^{+}=\hat{x}_{M}^{+}$ as follows:

$$
\begin{aligned}
& \mathbb{P}\left[\mathcal{T}_{2}^{(\mathrm{C})} \mid x_{M}^{+}=\hat{x}_{M}^{+}\right] \\
& =\mathbb{P}\left[\min _{x \in\left[1-x_{M}^{+, \mathrm{L}} / \hat{x}_{M}^{+}, 1\right]} B_{2}^{\mathrm{L}}(x) \geq \xi \delta^{\prime} \mid \mathcal{B}_{2}^{\mathrm{L}}\right] \\
& =\mathbb{P}\left[\min _{x \in\left[0, x_{M}^{+\mathrm{L}} / \hat{x}_{M}^{+}\right]} \widetilde{B}_{2}^{\mathrm{L}}(x) \geq \xi \delta^{\prime} \mid \widetilde{\mathcal{B}}_{2}^{\mathrm{L}}\right] \\
& \geq 1-\frac{\xi \delta^{\prime}}{\xi}=1-\delta^{\prime},
\end{aligned}
$$

where:

- (162) follows from the definition of $B_{2}^{\mathrm{L}}$ in (148), which implies that $B_{2}^{\mathrm{L}}$ is non-negative and has $B_{2}^{\mathrm{L}}\left(1-\frac{x_{M}^{+} \mathrm{L}}{\hat{x}_{M}^{+}}\right)=$ $\xi$ (recall from the definition of $x_{M}^{+, \mathrm{L}}$ that $W_{x_{M}^{+, \mathrm{L}}}=M^{+}-$ $\left.\delta^{\prime} \sqrt{2 \pi}\right)$.

- 163 follows by (160). Note also that the condition $\min _{x \in\left[1-x_{M}^{+} \mathrm{L} / \hat{x}_{M}^{+}, 1\right]} B_{2}^{\mathrm{L}}(x)>0$ is directly inherited from $\min _{x \in[0,1]} B_{2}^{\mathrm{L}}(x)>0$;

- (164) follows from Lemma 7 (with $u=\xi$ and $\varepsilon=\delta^{\prime} \xi$ ).

Since $\mathbb{P}\left[\mathcal{T}_{2}^{(\mathrm{C})} \mid x_{M}^{+}=\hat{x}_{M}^{+}\right] \geq 1-\delta^{\prime}$ for all $\hat{x}_{M}^{+}$, we have $\mathbb{P}\left[\mathcal{T}_{2}^{(\mathrm{C})}\right] \geq 1-\delta^{\prime}$. Similarly, as $B_{2}^{\mathrm{L}}$ is equal in distribution to $B_{2}^{\mathrm{R}}, \mathbb{P}\left[\mathcal{T}_{2}^{(\mathrm{D})}\right] \geq 1-\delta^{\prime}$.

When all four events and $\mathcal{T}_{1}$ hold, if $r^{+}(x)$ is less or equal to $c_{6} \delta^{2} \sqrt{\Delta}$, then $r^{-}(x)$ is greater or equal to $c_{6} \delta^{2} \sqrt{\Delta}$, where $c_{6} \delta^{2} \sqrt{\Delta}=\left(\delta^{\prime}\right)^{2} \sqrt{2 \Delta}$ for $c_{6}=0.01 \sqrt{2}$ (and vice versa). By the union bound and choice of $\delta^{\prime}=0.1 \delta$,

$$
\begin{aligned}
\mathbb{P}\left[\mathcal{T}_{2}\right] & \geq \mathbb{P}\left[\mathcal{T}_{2}^{(\mathrm{A})} \cap \mathcal{T}_{2}^{(\mathrm{B})} \cap \mathcal{T}_{2}^{(\mathrm{C})} \cap \mathcal{T}_{2}^{(\mathrm{D})}\right] \\
& \geq 1-\Delta^{\eta}-2\left(\delta^{\prime}\right)^{2}-\Delta^{\eta}-2\left(\delta^{\prime}\right)^{2}-\delta^{\prime}-\delta^{\prime} \\
& \geq 1-2 \Delta^{\eta}-\delta .
\end{aligned}
$$

Bounding $\mathbb{P}\left[\mathcal{T}_{3}\right]$. Recall the definitions of $\eta_{\delta}(\cdot)$ and $\alpha_{\delta}(\cdot)$ in 10$]$. Divide the time horizon $[-\Delta, 1+\Delta]$ into a time grid of size $\Delta$, with grid points $\tau_{k} \triangleq \min (k \Delta, 1+\Delta)$ for all $-1 \leq k \leq\lceil 1 / \Delta\rceil+1$ and intervals $I_{k} \triangleq\left[\tau_{k}, \tau_{k+1}\right]$ for all $-1 \leq k \leq\lceil 1 / \Delta\rceil$. For each interval, we define the running maximum and running minimum as follows:

$$
\tilde{W}_{I_{k}}^{\max } \triangleq \max _{x \in I_{k}} \tilde{W}_{x}, \quad \tilde{W}_{I_{k}}^{\min } \triangleq \min _{x \in I_{k}} \tilde{W}_{x} .
$$

We define the event $\mathcal{S} \triangleq \mathcal{S}_{1} \cap \mathcal{S}_{2} \cap \mathcal{S}_{3}$, where $\mathcal{S}_{i}$ for $i=1,2,3$ 
are defined as follows:

$$
\begin{aligned}
& \mathcal{S}_{1} \triangleq \bigcap_{k=-1}^{\lceil 1 / \Delta\rceil}\left\{\tilde{W}_{I_{k}}^{\max } \leq \max \left(\tilde{W}_{\tau_{k}}, \tilde{W}_{\tau_{k+1}}\right)+\eta_{\Delta}(\Delta)\right\} \\
& \mathcal{S}_{2} \triangleq \bigcap_{k=-1}^{\lceil 1 / \Delta\rceil}\left\{\tilde{W}_{I_{k}}^{\min } \geq \min \left(\tilde{W}_{\tau_{k}}, \tilde{W}_{\tau_{k+1}}\right)-\eta_{\Delta}(\Delta)\right\} \\
& \mathcal{S}_{3} \triangleq \bigcap_{k=-1}^{\lceil 1 / \Delta\rceil}\left\{\left|\tilde{W}_{\tau_{k}}-\tilde{W}_{\tau_{k+1}}\right| \leq \alpha_{\Delta}(\Delta)\right\} .
\end{aligned}
$$

We first show that $\mathcal{S}$ implies $\mathcal{T}_{3}$ (see Definition 3). Suppose that $\mathcal{S}$ holds and there exists an integer $-1 \leq k \leq\lceil 1 / \Delta\rceil-2$ such that $x-\Delta$ falls into the $k$-th interval $I_{k}$ while $x+\Delta$ falls into the $(k+2)$-nd interval $I_{k+2}$. Then, the difference $\mid \tilde{W}_{x-\Delta}-$ $\tilde{W}_{x+\Delta} \mid$ can be upper bounded via the triangle inequality:

$$
\begin{aligned}
& \quad\left|\tilde{W}_{x-\Delta}-\tilde{W}_{x+\Delta}\right| \\
& =\max \left(\tilde{W}_{x-\Delta}-\tilde{W}_{x+\Delta}, \tilde{W}_{x+\Delta}-\tilde{W}_{x-\Delta}\right) \\
& \leq \max \left(\tilde{W}_{I_{k}}^{\max }-\tilde{W}_{I_{k+2}}^{\min }, \tilde{W}_{I_{k+2}}^{\max }-\tilde{W}_{I_{k}}^{\min }\right) \\
& \leq \max \left[\max \left(\tilde{W}_{\tau_{k}}, \tilde{W}_{\tau_{k+1}}\right)\right. \\
& \quad-\min \left(\tilde{W}_{\tau_{k+2}}, \tilde{W}_{\tau_{k+3}}\right)+2 \eta_{\Delta}(\Delta), \\
& \quad \max \left(\tilde{W}_{\tau_{k+2}}, \tilde{W}_{\tau_{k+3}}\right) \\
& \left.\quad-\min \left(\tilde{W}_{\tau_{k}}, \tilde{W}_{\tau_{k+1}}\right)+2 \eta_{\Delta}(\Delta)\right] \\
& \leq 3 \alpha_{\Delta}(\Delta)+2 \eta_{\Delta}(\Delta) \\
& \leq c_{4} \sqrt{\Delta \ln (1 / \Delta)},
\end{aligned}
$$

where (173) follows from the definitions of running maximum and minimum, 174 follows from events $\mathcal{S}_{1}$ and $\mathcal{S}_{2}$, 175 follows from event $\mathcal{S}_{3}$, and (176) uses the definitions of $\eta_{\delta}(\cdot)$ and $\alpha_{\delta}(\cdot)$ in 10 .

For any $0 \leq x \leq 1$, the absolute difference between the two regret functions can be expressed as the difference between the $\tilde{W}$ values corresponding to two $2 \Delta$-separated points:

$$
\begin{aligned}
\left|r^{+}(x)-r^{-}(x)\right| & =\left|\left(W_{x_{M}^{+}}^{+}-W_{x}^{+}\right)-\left(W_{x_{M}^{-}}^{-}-W_{x}^{-}\right)\right| \\
& =\left|M^{+}-M^{-}-W_{x}^{+}+W_{x}^{-}\right| \\
& =\left|\tilde{W}_{x-\Delta}-\tilde{W}_{x+\Delta}\right| \\
& \leq c_{4} \sqrt{\Delta \ln (1 / \Delta)}
\end{aligned}
$$

where $(178)$ follows from the definitions of $x_{M}^{+}$and $x_{M}^{-}$in (54), (179) follows from definitions of $W^{+}$and $W^{-}$in (51) and (52), and (180) follows from (176) (which holds uniformly in $x$ ). Hence, the absolute difference between the two regret functions is upper bounded by $\mathcal{O}(\sqrt{\Delta \ln (1 / \Delta)})$ everywhere.

It remains to show that $\mathcal{S}$ holds with probability at least $1-\Delta$. From Lemma A.5 in Appendix A, each sub-event of $\mathcal{S}_{1}$ and $\mathcal{S}_{2}$ holds with probability at least $1-(\Delta \cdot \Delta)^{5}$. By the union bound over the $\lceil 1 / \Delta\rceil$ many sub-events of $\mathcal{S}_{1}$, we have $\mathbb{P}\left[\mathcal{S}_{1}\right] \geq 1-\Delta^{9}$. Similarly, we have $\mathbb{P}\left[\mathcal{S}_{2}\right] \geq 1-\Delta^{9}$.

Similar to the argument for lower bounding the probability of $\mathcal{M}_{1}$ in the proof of Lemma 2 each sub-event of $\mathcal{S}_{3}$ holds with probability at least $1-(\Delta \cdot \Delta)^{3}$ by Lemma 1 . Hence, we can lower bound $\mathbb{P}\left[\mathcal{S}_{3}\right]$ by $1-\Delta^{5}$, and by the union bound on $\mathcal{S}_{1}, \mathcal{S}_{2}$ and $\mathcal{S}_{3}$, we have

$$
\mathbb{P}\left[\mathcal{T}_{3}\right] \geq \mathbb{P}[\mathcal{S}] \geq 1-\Delta
$$

as event $\mathcal{S}$ implies $\mathcal{T}_{3}$.

Lower bounding $\mathbb{P}[\mathcal{T}]$. Recall from Section VII-E that $\mathbb{P}\left[\mathcal{T}_{1}\right] \geq 1-\delta_{1}=1-\Delta^{\eta}$. Combining this with the lower bounds on $\mathbb{P}\left[\mathcal{T}_{2}\right]$ and $\mathbb{P}\left[\mathcal{T}_{3}\right]$, and using the union bound on the events $\mathcal{T}_{1}, \mathcal{T}_{2}$ and $\mathcal{T}_{3}$, we have

$$
\mathbb{P}[\mathcal{T}] \geq 1-3 \Delta^{\eta}-\delta-\Delta
$$

as desired.

\section{F. Proof of Lemma 9 (Regret Bound Using Fano's Inequality)}

By Markov's inequality, we have

$$
\mathbb{E}_{\tilde{w}}\left[r_{T}\right] \geq c_{3} \delta^{2} \sqrt{\Delta} \cdot \mathbb{P}_{\tilde{w}}\left[r_{T} \geq c_{3} \delta^{2} \sqrt{\Delta}\right] .
$$

By Definition 3, conditioned on $\tilde{W}=\tilde{w}$ satisfying $\mathcal{T}, r_{T}$ is smaller than $c_{3} \delta^{2} \sqrt{\Delta}$ for at most one of the functions $W^{+}$ and $W^{-}$. Hence, if we let $\hat{V}$ denote the index in $\{+,-\}$ corresponding to the smaller regret, we find that if the regret associated with $v$ is smaller than $c_{3} \delta^{2} \sqrt{\Delta}$, we must have $\hat{V}=$ $v$. Therefore,

$$
\mathbb{P}_{\tilde{w}}^{v}\left[r_{T} \geq c_{3} \delta^{2} \sqrt{\Delta}\right] \geq \mathbb{P}_{\tilde{w}}^{v}[\hat{V}=v],
$$

where the superscript indicates conditioning on $V=v$. Hence, we can lower bound the above probability as follows, using the fact that $V$ is equiprobable on $\{+,-\}$ :

$$
\begin{aligned}
\mathbb{P}_{\tilde{w}}\left[r_{T} \geq c_{3} \delta^{2} \sqrt{\Delta}\right] & =\frac{1}{2} \sum_{v \in\{+,-\}} \mathbb{P}_{\tilde{w}}^{v}\left[r_{T} \geq c_{3} \delta^{2} \sqrt{\Delta}\right] \\
& \geq \frac{1}{2} \sum_{v \in\{+,-\}} \mathbb{P}_{\tilde{w}}^{v}[\hat{V}=v] \\
& \geq H_{2}^{-1}\left(\log 2-I_{\tilde{w}}(V ; \mathbf{x}, \mathbf{y})\right),
\end{aligned}
$$

where (186) follows from events $\mathcal{T}_{1}$ and $\mathcal{T}_{2}$ in Definition 3 and 187) follows from the binary version of Fano's inequality, e.g., as stated in [24, Remark 1].

Supplementary material (appendix). The supplementary material is uploaded as a separate document, containing Appendix A stating known properties of Brownian motion.

Acknowledgment. The authors gratefully acknowledge Prof. Rongfeng Sun for helpful discussions regarding properties of Brownian motion.

\section{REFERENCES}

[1] T. S. Kuhn, Black-Body Theory and the Quantum Discontinuity, 1894 1912. University of Chicago Press, 1987.

[2] J. Adler, I.-M. Sintorn, R. Strand, and I. Parmryd, "Conventional analysis of movement on non-flat surfaces like the plasma membrane makes Brownian motion appear anomalous," Communications Biology, vol. 2, no. 1, p. 12, 2019.

[3] M. Kijima, Stochastic Processes with Applications to Finance. Chapman and Hall/CRC, 2016. 
[4] J.-B. Grill, M. Valko, and R. Munos, "Optimistic optimization of a Brownian," in Advances in Neural Information Processing Systems, 2018, pp. 3005-3014.

[5] H. Al-Mharmah and J. M. Calvin, "Optimal random non-adaptive algorithm for global optimization of Brownian motion," Journal of Global Optimization, vol. 8, no. 1, pp. 81-90, 1996.

[6] M. Abdechiri, M. R. Meybodi, and H. Bahrami, "Gases Brownian motion optimization: An algorithm for optimization (GBMO)," Applied Soft Computing, vol. 13, no. 5, pp. 2932-2946, 2013.

[7] J. M. Calvin, M. Hefter, and A. Herzwurm, "Adaptive approximation of the minimum of Brownian motion," Journal of Complexity, vol. 39, pp. 17-37, 2017.

[8] J. Mockus, Bayesian Approach to Global Optimization: Theory and Applications. Springer, 1989.

[9] N. Srinivas, A. Krause, S. M. Kakade, and M. W. Seeger, "Informationtheoretic regret bounds for Gaussian process optimization in the bandit setting," IEEE Transactions on Information Theory, vol. 58, no. 5, pp. 3250-3265, 2012.

[10] J. Scarlett, "Tight regret bounds for Bayesian optimization in onedimension," International Conference on Machine Learning, 2018.

[11] S. Du, "Gradient descent for non-convex problems in modern machine learning," Ph.D. dissertation, Carnegie Mellon University, 2019.

[12] R. Marchant and F. Ramos, "Bayesian optimisation for intelligent environmental monitoring," in 2012 IEEE/RSJ International Conference on Intelligent Robots and Systems, 2012, pp. 2242-2249.

[13] K. Swersky, J. Snoek, and R. P. Adams, "Freeze-thaw Bayesian optimization," 2014, https://arxiv.org/abs/1406.3896.

[14] S. Grünewälder, J.-Y. Audibert, M. Opper, and J. Shawe-Taylor, "Regret bounds for Gaussian process bandit problems," in International Conference on Artificial Intelligence and Statistics, 2010, pp. 273-280.

[15] K. Kawaguchi, L. P. Kaelbling, and T. Lozano-Pérez, "Bayesian optimization with exponential convergence," in Advances in Neural Information Processing Systems, 2015, pp. 2809-2817.

[16] R. Munos, "Optimistic optimization of a deterministic function without the knowledge of its smoothness," in Advances in Neural Information Processing Systems, 2011, pp. 783-791.

[17] X. Shang, E. Kaufmann, and M. Valko, "General parallel optimization a without metric," in Algorithmic Learning Theory, 2019, pp. 762-788.

[18] O. Shamir, "On the complexity of bandit and derivative-free stochastic convex optimization," in Conference on Learning Theory, 2013, pp. 324.

[19] M. Raginsky and A. Rakhlin, "Information-based complexity, feedback and dynamics in convex programming," IEEE Transactions on Information Theory, vol. 57, no. 10, pp. 7036-7056, 2011.

[20] I. Karatzas and S. E. Shreve, Brownian Motion and Stochastic Calculus. Springer-Verlag, 1988.

[21] T. Lattimore and C. Szepesvári, Bandit algorithms. Cambridge University Press, 2020

[22] N. de Freitas, M. Zoghi, and A. J. Smola, "Exponential regret bounds for Gaussian process bandits with deterministic observations," in International Conference on Machine Learning, 2012.

[23] R. Durett, D. Iglehart, and D. Miller, "Weak convergence to Brownian meander and Brownian excursion," The Annals of Probability, vol. 5, no. 1 , pp. $117-129,1977$

[24] J. Scarlett and V. Cevher, "An introductory guide to Fano's inequality with applications in statistical estimation," 2019, https://arxiv.org/abs/1901.00555.

[25] F. Iafrate and E. Orsingher, "Some results on the Brownian meander with drift," Journal of Theoretical Probability, pp. 1-27, 2019.

[26] O. Kallenberg, Foundations of Modern Probability. S Springer Science \& Business Media, 2006.

[27] I. Denisov, "Random walk and the Wiener process considered from a maximum point," Teor. Veroyatnost. i Primenen, vol. 28, no. 4, pp. 785788,1983

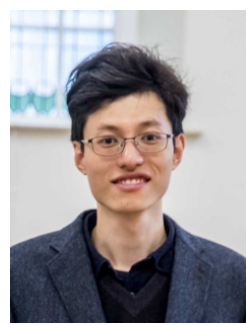

Zexin Wang received the Bachelor of Science degree in Quantitative Finance from National University of Singapore, Singapore, in 2019. He is currently a Ph.D. student with the Mathematical Finance section at Department of Mathematics, Imperial College London, London, U.K. From May 2019 to July 2019, he was a research assistant with the Department of Computer Science, National University of Singapore. His research interests are in the area of mathematical finance, mainly market microstructure, derivative pricing, limit order zoning and liquidation

costs.

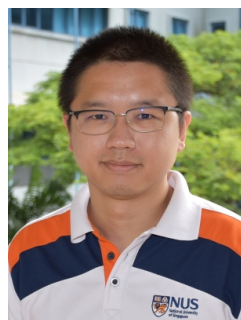

Vincent Y. F. Tan (S'07-M'11-SM'15) received the B.A. and M.Eng. degrees in electrical and information sciences from Cambridge University, Cambridge, U.K., in 2005, and the Ph.D. degree in electrical engineering and computer science (EECS) from the Massachusetts Institute of Technology (MIT), Cambridge, MA, USA, in 2011. He is currently an Associate Professor with the Department of Electrical and Computer Engineering and the Department of Mathematics, National University of Singapore. His research interests include information theory, machine learning, and statistical signal processing. He is currently an Associate Editor for the IEEE TRANSACTIONS ON SIGNAL PROCESSING and an Associate Editor of machine learning for the IEEE TRANSACTIONS ON INFORMATION THEORY.

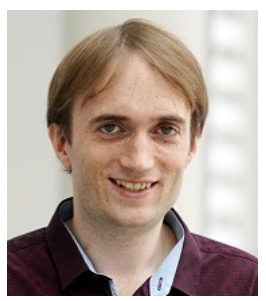

Jonathan Scarlett (S'14-M'15) received the B.Eng. degree in electrical engineering and the B.Sci. degree in computer science from the University of Melbourne, Australia. From October 2011 to August 2014, he was a Ph.D. student in the Signal Processing and Communications Group at the University of Cambridge, United Kingdom. From September 2014 to September 2017, he was postdoctoral researcher with the Laboratory for Information and Inference Systems at the École Polytechnique Fédérale de Lausanne, Switzerland. Since January 2018, he has been an assistant professor in the Department of Computer Science and Department of Mathematics, National University of Singapore. His research interests are in the areas of information theory, machine learning, signal processing, and high-dimensional statistics. He received the Singapore National Research Foundation (NRF) fellowship, and the NUS Presidential Young Professorship. 


\title{
Supplementary Material \\ Tight Regret Bounds for Noisy Optimization of a Brownian Motion
}

\author{
Zexin Wang, Vincent Tan, and Jonathan Scarlett
}

APPENDIX A

\section{RESUlts CONCERning Brownian Motion}

Lemma A.1 (Karatzas and Shreve [20, Problem 8.2]). Let $W_{x}$ be a Brownian motion and $M_{x}=\max _{0 \leq x^{\prime} \leq x} W_{x^{\prime}}$. The probability density function of $M_{x}$ satisfies

$$
\mathbb{P}\left[M_{x} \in \mathrm{d} b\right]=\mathbb{P}\left[\left|W_{x}\right| \in \mathrm{d} b\right] .
$$

Lemma A.2 (Grill et al. [4. Proof of Lemma 1]). Given the values of a Brownian motion at the ends of an interval [a,b] being $w_{a}$ and $w_{b}$, the cumulative distribution function of the running maximum of the Brownian motion in the interval $[a, b]$ is, for all $y \geq \max \left(w_{a}, w_{b}\right)$,

$$
\mathbb{P}\left[\max _{x \in[a, b]} W_{x}>y \mid W_{a}=w_{a}, W_{b}=w_{b}\right]=\exp \left[-\frac{2\left(y-w_{a}\right)\left(y-w_{b}\right)}{b-a}\right] .
$$

Lemma A.3 (Kallenberg [26, Equation (3.1)]). Let B be a Brownian motion with initial value $u$ ? the joint distribution function of it and its running minimum for any $y>v, u>v, s>0$ is as follows:

$$
\begin{aligned}
& \mathbb{P}\left[B(s) \in \mathrm{d} y, \min _{0 \leq z \leq s} B(z)>v \mid B(0)=u\right] \\
& =\left[\exp \left(-\frac{(y-u)^{2}}{2 s}\right)-\exp \left(-\frac{(2 v-y-u)^{2}}{2 s}\right)\right] \frac{\mathrm{d} y}{\sqrt{2 \pi s}} .
\end{aligned}
$$

Lemma A.4 (Denisov [27, Theorem 1]). Let $W$ be a standard Brownian motion with maximizer $x_{M}$ and maximum value $M=\max _{x \in D} W_{x}$. If we define $B_{2}^{\mathrm{L}}$ and $B_{2}^{\mathrm{R}}$ as

$$
B_{2}^{\mathrm{L}}(x) \triangleq \frac{M-W_{x_{M}-x \cdot x_{M}}}{\sqrt{x_{M}}}, \quad \text { and } \quad B_{2}^{\mathrm{R}}(x) \triangleq \frac{M-W_{x_{M}+x \cdot\left(1-x_{M}\right)}}{\sqrt{1-x_{M}}},
$$

then $B_{2}^{\mathrm{L}}$ and $B_{2}^{\mathrm{R}}$ are independent and identically distributed Brownian meanders (i.e., BM conditioned on being positive), and they are also independent of $x_{M}$.

Lemma A.5 (Grill et al. [4, Proof of Lemma 1]). We have the following high-probability upper bound on the running maximum of a Brownian motion in the interval $[a, b]$ :

$$
\mathbb{P}\left[\sup _{x \in[a, b]} W_{x}>\max \left(W_{a}, W_{b}\right)+\eta_{\delta}(b-a)\right] \leq[\delta(b-a)]^{5},
$$

with $\eta_{\delta}(\cdot)$ defined in 10 .

${ }^{9}$ In [26] a possible drift is included, but for our purposes a drift of zero suffices. 